\title{
PHILelergite
}

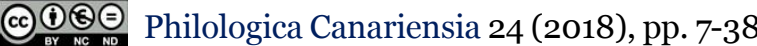

DOI: 10.20420/PhilCan.2018.195

Recibido: 19 de julio de 2017; aceptado: 6 de marzo de 2018

Publicado: 18 de julio de 2018

\section{LA LITERATURA COMO MANIFESTACIÓN DEL LENGUAJE. ANÁLISIS COMPARATIVO DE LA SINTAXIS DE DOS CUENTOS}

\author{
ISAMARY ALDAMA PANDO \\ Centro Cultural Pablo de la Torriente Brau, La Habana
}

\begin{abstract}
RESUMEN: A finales de los ochenta, como parte del proyecto de investigación "El español en Cuba" se llevó a cabo el estudio "Sintaxis de la norma culta escrita en Cuba: cuento y artículo periodístico", bajo la dirección del profesor Luis Enrique Rodríguez. Entre sus objetivos estaba aplicar el análisis sintáctico a la caracterización linguoestilística de textos más extensos. Los resultados finales de la investigación nunca fueron publicados, pero la metodología elaborada es aún empleada en cursos de posgrado impartidos en la Facultad de Artes y Letras de la Universidad de La Habana. En el presente trabajo se aplica la metodología propuesta, con el fin de analizar dos cuentos y compararlos. La intención no es realizar un análisis linguoestilístico detallado, sino mostrar la aplicación de un método de análisis sintáctico a un rasgo particular en el estilo de los cuentos: la técnica del flujo de conciencia.
\end{abstract}

PALABRAS CLAVE: sintaxis, estilística, literatura, lenguaje

Literature as a manifestation of language.

Comparative analysis of the syntax of two tales

\begin{abstract}
In the late eighties, as part of the research project "The Spanish language in Cuba", the study "Syntax of the educated norm of Cuba: the short story and newspaper article" was performed under the direction of Professor Luis Enrique Rodríguez. One of the objectives was to apply syntactic analysis to the linguo-stylistic characterization of more extensive texts. The final results of the investigation were never published, but the methodology developed is still used in postgraduate courses taught at the Faculty of Arts and Letters of the University of Havana. In the present article, the proposed methodology is applied to analyze two short stories and compare them. Our aim is not to make a detailed linguo-stylistic analysis, but to show how a method of syntactic analysis may be applied to a particular feature in the style of the stories: the technique of the stream of consciousness. KEYWORDS: syntax, stylistics, literature, language
\end{abstract}

\section{INTRODUCCIÓN}

La literatura, como situación comunicativa entre un autor y un lector, es uno de los modos en que se manifiesta el lenguaje: "las formas lingüísticas se ponen en funcionamiento para construir formas de comunicación y de representación del mundo - real o imaginario-" (Calsamiglia y Tusón, 1999, 15). El texto escrito es entonces el instrumento con el que se 
materializa y transmite el pensamiento del autor/enunciador ${ }^{1}$ y que permite la realización del proceso interior del pensamiento del lector por medio de la decodificación del mensaje.

Este vínculo estrecho entre pensamiento y lenguaje que se manifiesta en la literatura tiene una de sus expresiones en la técnica del flujo de conciencia, donde el "autor está obligado a representar el caos y los accidentes de la mente, de la conciencia, sin patrón, sin disciplina ni claridad" (Burunat, 1980, 24). Para ello, en el lenguaje literario se utilizan con frecuencia determinadas estructuras lingüísticas -fundamentalmente en el nivel sintáctico- que posibilitan definir el empleo de un estilo propio del flujo del pensamiento. Es necesario entonces analizar las características de un texto específico para así determinar cuáles serían esas constantes que permitirían reconocer que se está en presencia de la mencionada técnica narrativa. Por esta razón he escogido el cuento "Elisa" (2009) de David Camps, que, aunque no sería el ejemplo "más feliz" para caracterizar el estilo del flujo del pensamiento, sí tiene algunas características que podrían ser destacables, por lo que, después de hacer el análisis sintáctico de este texto y determinar qué elementos lo caracterizan en cuanto al empleo de la técnica del flujo de conciencia, se comparará con el cuento "Miedo" (2009) de Pedro Juan Gutiérrez, en el cual no se persigue la imitación del monólogo interior. Para cumplir los objetivos propuestos se partirá de la aplicación del método de análisis creado en Cuba por el profesor Luis Enrique Rodríguez Suárez.

\section{ANTECEDENTES}

Los primeros pasos de la estilística en su condición de ciencia se dan como estudios de un hecho particular del lenguaje: la Estilística latina de Berger se centraba en el estudio de locuciones particulares (apud Yllera, 1979, 13-14), mientras Bally (1905, 1909) se interesaba en los medios de expresión disponibles en una lengua. El lingüista suizo partía de la distinción de tres dimensiones de la estilística, que correspondían al estudio de los medios expresivos del lenguaje general, de una lengua concreta o del habla de un individuo determinado, con lo cual hacía referencia a una estilística general, una colectiva y otra individual, pero definiéndola en sentido general como una ciencia que estudia "les faits d'expression du langage organisé au point de vue de leur contenu affectif, c'est-à-dire l'expression des faits de la sensibilité par le langage et l'action des faits de langage sur la sensibilité" (Bally, 1909, 16). . Pese a ello, solo le interesó la segunda y a ella se dedicó en su Traité de stylistique française, centrándose en el aspecto afectivo de la lengua y, por tanto, dejando el lenguaje literario fuera de los estudios estilísticos. La negativa del ginebrino a considerar la posibilidad del análisis estilístico de la literatura no fue seguida por sus discípulos (cf. Marouzeau, 1959; Bruneau, 1970 [1951]; Cressot, 1969 [1947]), pues, según se vio posteriormente, sus hallazgos podrían aplicarse a cualquier estudio literario.

Así, en la escuela alemana, Vossler $(1929,1955,1963)$ y Spitzer $(1931,1968)$ reconocen la existencia de una estilística dedicada al estudio de la lengua y otra al estudio del lenguaje poético (o literario), pero subordinan el análisis al aspecto estético y afectivo, sin ignorar el lingüístico. Sin embargo, el primero está más apegado al historicismo en tanto le interesa cómo el autor se forja su propio estilo dentro de un lenguaje históricamente determinado (Yllera, 1979, 20). Mientras, Spitzer da primacía a la expresión individual del autor, partiendo del postulado de que, a través de las peculiaridades lingüísticas que manifiestan los textos, es 
posible determinar las características del contexto histórico-social que rodea al autor en el momento de la creación.

En el ámbito hispano, Amado Alonso (1955) y Dámaso Alonso (1950) también definen dos direcciones de estudio: una dedicada a la lengua corriente (la estilística de la lengua que defendía Bally) y otra a la lengua de la poesía (la estilística del habla, que comprende la literaria, ignorada por Bally). Y no hacen distinción entre ambas, pues, como indica Amado Alonso, "la estilística, como ciencia de los estilos literarios, tiene como base a esa otra estilística que estudia el lado afectivo, activo, imaginativo y valorativo de las formas de hablar fijadas en el idioma" $(1955,81)$.

Asimismo, paralelo a estos acercamientos a la literatura se desarrolló una estilística de tipo cuantitativo. En ella se analizan estadísticamente las características de estilo y se elaboran perfiles estilísticos de textos a partir de datos estadísticos (Milic, 1967; Doležel y Bailey, 1969).

La disciplina de la crítica lingüística constituye otra contribución de los estudios lingüísticos a la sociología del lenguaje, ya que el análisis del texto se concentra en la relación entre las decisiones lingüísticas del hablante y el mundo social (cf. Fowler, Hodge, Kress, Trew, 1979; Hodge y Kress, 1979). Cualquier tipo de texto puede ser objeto de análisis de esta disciplina, si bien los críticos lingüísticos centran su interés en el estudio de textos literarios (cf. Davie, 1966; Baker, 1967; Leech, 1969 y 1976; Fowler, 1971, 1981 y 1986). Entre ellos habría que destacar a Geoffrey Leech y Robert Fowler, cuyos trabajos han contribuido enormemente a la incorporación de métodos lingüísticos a los estudios literarios.

Según Leech, en los textos literarios "every feature of language is a matter of design rather than chance or carelessness" $(1969,220), 3$ por lo que es objetivo de la crítica lingüística descubrir cuáles son las relaciones que se establecen entre las estructuras y niveles de la literatura. Mientras, Fowler plantea que la crítica puede mejorarse mediante el conocimiento del lenguaje $(1971,11)$ y que la terminología lingüística puede contribuir a la crítica al proporcionarle un metalenguaje en lo concerniente al lenguaje mismo (1971, 12). Al respecto, es interesante cómo, por medio de ejemplos tomados de la narrativa, la poesía y el teatro, Fowler muestra las estructuras comunes al lenguaje literario y al ordinario, ya que para llevar a cabo el proceso de lectura es necesaria la competencia lingüística que el lector pone en práctica cotidianamente.

Sin embargo, lo propuesto por la crítica lingüística no es algo nuevo, pues, según Wellek, esta corriente se remonta al formalismo ruso y tuvo su origen entre "positivistas con un ideal científico de erudición literaria" (citado en Pretorius, 1981, 2), lo que queda demostrado en palabras de Roman Jakobson, quien es considerado por George Steiner "el gran mediador entre literatura y gramática” (citado en Arkins, 1994, 3). Con respecto a la relación entre lenguaje y literatura, decía el teórico ruso:

If there are some critics who still doubt the competence of linguistics to embrace the field of poetics, I privately believe that the poetic in competence of some bigoted linguists has been mistaken for an inadequacy of the linguistic science itself. [...] a linguist deaf to the poetic function of language and a literary scholar indifferent to linguistic problems and unconversant with linguistic methods are equally flagrant anachronisms. (1960, 377) 4

Con lo cual planteaba la necesidad de considerar a la lingüística ciencia rectora del estudio de todas las manifestaciones del lenguaje, incluyendo la literatura. 
A partir de los planteamientos del formalismo ruso, y su enfoque ontológico del texto literario, investigadores y teóricos de la literatura y la lingüística prestaron mayor atención al lenguaje literario. Así, es posible encontrar, por ejemplo, estudios en los que se considera la obra literaria acto de habla y como tal se analiza, aplicando la pragmática al discurso literario (Lecercle, 1999; Ohmann, 1971; Pratt, 1977).

Tal como apunta Roger D. Sell (2000), en la obra literaria el autor imitará los actos de habla tal como los interpretan sus personajes. A lo cual podríamos añadir que no solo se haría con respecto a la imitación del diálogo real en el nivel intradiegético, sino que podría considerarse el hecho literario en sí mismo como un acto de habla en el que el autor tiene la intención de comunicarse con el lector. Es por ello por lo que es posible identificar el uso de las máximas de Grice (1975) no solo en la interacción entre los personajes, sino en lo dicho (o no dicho) por el autor (cf. Aldama, 2017), o analizar el texto literario tomando como punto de partida las ideas de Austin (1962) y Searle (1969) o la teoría de la relevancia de Sperber y Wilson (1986), en la que se incluyen las cuatro máximas de cooperación de Grice, o estudios de la inferencia en obras de ficción (cf. Cook, 1994; Kearns, 1999; Sell, 2000).

Han surgido, entonces, muy diversas disciplinas con el propósito de estudiar la obra literaria no solo como ente aislado, o sea, con un enfoque inmanente, sino en su relación con el exterior, ya sea buscando cómo el lenguaje empleado refleja el contexto de su creación; los modos en que el autor se relaciona con el lector; las relaciones de poder presentes en la obra; el tratamiento dado a la figura de la mujer; cómo el enunciador (el propio autor, el narrador o determinado personaje), por medio del lenguaje, refleja la imagen que tiene de sí mismo o de los otros, e innumerables tópicos de análisis que vinculan la teoría literaria, la lingüística, la semiótica, la filosofía del lenguaje, la psicología, la sociología y muchas otras disciplinas de las ciencias humanísticas.

Si bien el estudio que aquí se propone tiene sus bases en las corrientes lingüísticas mencionadas, mi intención no es hacer un análisis linguoestilístico detallado, sino aplicar un método de análisis sintáctico en función de la caracterización de un hecho estilístico concreto. Por tal razón, puede que haya algunos aspectos del análisis que no sean mencionados aquí. Ni tampoco se ha hecho una presentación minuciosa de las teorías precedentes, pues, para los efectos del estudio que se presenta, es mucho más importante la explicación del método en el que se basa.

A finales de los ochenta, como parte del proyecto de investigación "El español en Cuba" se llevó a cabo el estudio "Sintaxis de la norma culta escrita en Cuba: cuento y artículo periodístico", bajo la dirección del profesor Luis Enrique Rodríguez (1989). Entre los objetivos propuestos estaba realizar el análisis sintáctico de una muestra de 100 artículos de 10 periodistas, con temática fundamentalmente sociocultural, y cuentos de 10 narradores, que en su extensión abarcaran el espacio de los 100 artículos. Luego, se realizaría un estudio comparativo con la intención de determinar las características sintácticas y estilísticas de cada género, así como la correspondencia entre la forma y el contenido de los textos. Los resultados finales de la investigación nunca fueron publicados, pero la metodología elaborada es aún hoy empleada en cursos de posgrado impartidos en la Facultad de Artes y Letras de la Universidad de La Habana.

El método de análisis creado por Rodríguez se basa en el sistema de notación sintáctica desarrollado por la Dra. Ofelia García Cortiñas 5 (conocido como ESTRUCTURA BÁSICA COMPLEJA), aplicado en las universidades de La Habana, Las Villas y Santiago de Cuba para el 
análisis sintáctico de fragmentos de textos. Para ello se simbolizaba cada ORACión GRAMATICAL ${ }^{6}$ mediante un cuadro, cada uno de los cuales era ordenado de manera horizontal (según su orden de aparición) o vertical (según el nivel de jerarquía en la oración). Asimismo, también se reflejaban los nexos que relacionaban cada oración gramatical que componía una ORACIÓN PSICOLÓGICA.7 Veamos un ejemplo:

IAlgo ocurre y IIno puedo imaginar IIIqué puede ser.

Como muestran los números romanos, esta oración psicológica la componen tres oraciones gramaticales, es decir, tres estructuras sintácticas, cada una con su sujeto y su predicado, lo que queda reflejado gráficamente del siguiente modo:

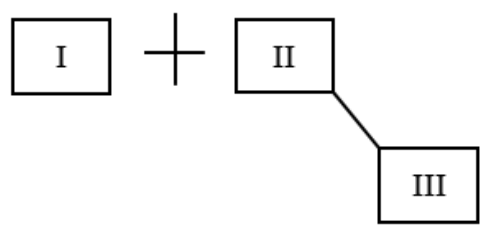

En esta representación gráfica, el signo + simboliza la relación de coordinación copulativa ${ }^{8}$ que se establece entre las oraciones I y II, y la línea diagonal que une a los otros dos cuadros simboliza la relación de subordinación entre las oraciones II y III, donde la primera es la regente y la segunda la subordinada.

En el sistema creado por Rodríguez para el estudio de la sintaxis de la norma culta cubana, la noción de ORACIÓN PSICOLÓGICA es sustituida por la de CONJUNTO ORACIONAL (CO): toda oración no subordinada, esté integrada por una oración simple (CO simple) o por una regente y una o varias subordinadas (CO complejo) (Rodríguez Suárez, 1989, 168). Asimismo, en aras de obtener mayor información sobre la oración analizada, el cuadro que simbolizaba la oración gramatical se subdividió en siete partes:

\begin{tabular}{|c|c|c|}
\hline $\mathrm{A}$ & $\mathrm{B}$ & $\mathrm{C}$ \\
\hline $\mathrm{D}$ & $\mathrm{E}$ & $\mathrm{F}$ \\
\hline \multicolumn{3}{|c|}{$\mathrm{G}$} \\
\hline
\end{tabular}
A: nivel ocupado por la oración (siendo el número 1 el que marca la oración regente)
B: orden de aparición de la oración dentro del CO
C: en caso de ser subordinada, número de la oración a la que se subordina
D: precisa si la oración es bimembre (b) o unimembre (u)
E: precisa la índole del núcleo predicativo (si es verbo conjugado [v], gerundio [g], oración subordinada [or], sustantivo [s], etc.) 9
F: precisa la función desempeñada por la oración subordinada
G: nexo que establece la relación de subordinación

Así, en el ejemplo presentado anteriormente, tendríamos dos conjuntos oracionales: uno simple y otro complejo, integrados por una oración gramatical el primero, y el segundo por dos, y gráficamente se representaría del siguiente modo: ${ }^{10}$ 


\begin{tabular}{|c|c|c|}
\hline 1 & 1 & 0 \\
\hline $\mathrm{b}$ & $\mathrm{v}$ & 0 \\
\hline \multicolumn{3}{|c|}{0} \\
\hline
\end{tabular}

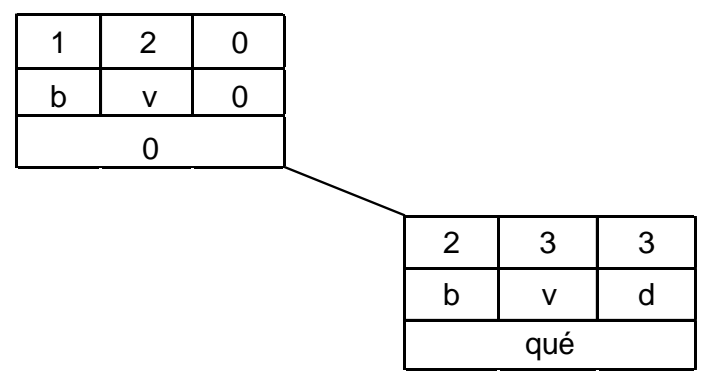

En los cuadros que representan las dos primeras oraciones, el cero (' 0 ') es empleado para marcar la ausencia de información en las casillas correspondientes al número de la oración a la que se subordinan, así como a la función de la subordinada, pues son oraciones regentes, de ahí que tampoco sea pertinente determinar el nexo que establece la relación de subordinación.

Sin embargo, el '0' también es empleado para marcar la interrupción de oraciones, como muestra el ejemplo propuesto por Rodríguez $(1989,172)$ :

${ }^{1 *}$ Luego, ${ }^{2}$ sabiendo que ${ }^{3}$ era inútil ${ }^{4}$ rogar a ${ }^{5}$ quien sueña ya con ${ }^{6}$ lo que hay detrás de los horizontes, 7 mi madre empezó a preguntarme, con voz dolorida, por la seguridad de las naves y la pericia de los pilotos.

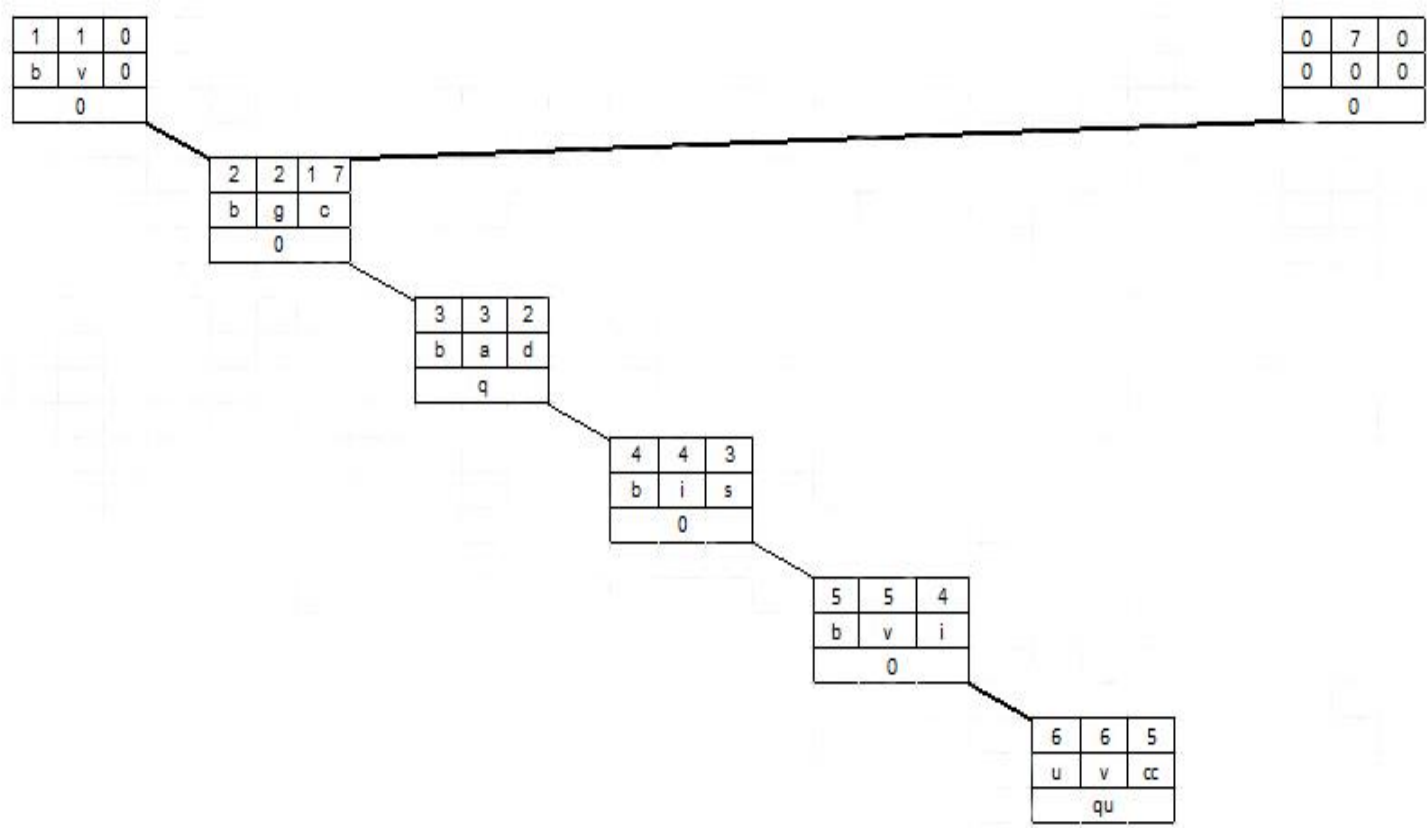

* En este nuevo sistema los números romanos son sustituidos por los arábigos, puesto que al analizar grandes cantidades de oraciones se haría muy complejo emplear el sistema romano, además de que el arábigo es el adecuado para obtener datos estadísticos. 
Nótese cómo en la oración 7 aparece el '0' para marcar la ausencia de información, al tiempo que sirve para señalar que esta oración es continuación de la primera, lo que permite, con solo ver la secuencia numérica, saber cuántas oraciones hay intercaladas en esa primera. Es por eso también por lo que en la oración 2, en la casilla correspondiente al número de la oración a la que se subordina, aparecen el 1 y el 7, pues ambas constituyen la oración subordinante.

Puesto que no es propósito de este texto hacer una presentación in extenso del método creado por el profesor Rodríguez, solo se mencionan los aspectos más destacados de su metodología, ya que, como puede verse, es muy compleja. Sin embargo, no debe dejar de mencionarse los innumerables beneficios que ofrece a la hora de analizar sintácticamente textos completos, sobre todo si se requieren datos estadísticos: permite trabajar más cómodamente con bases de datos, lo que posibilita determinar con rapidez la frecuencia de aparición de determinadas estructuras oracionales, nexos subordinantes, tipos de núcleo predicativo y muchos otros datos que permitirán a su vez determinar las ocurrencias y constantes que, unido al contenido semántico y la intencionalidad estética, caracterizan determinado género literario, discurso o a un autor, al tiempo que se compara con otros, tal y como se podrá comprobar a continuación en el análisis de los dos cuentos propuestos.

\section{ANÁLISIS DE LOS CUENTOS}

\section{1. "Elisa"}

\subsubsection{Caracterización}

El cuento narra la historia del último encuentro que tuvo el narrador-personaje ${ }^{11}$ con Elisa, su pareja. A partir de la información que se da en el texto se puede inferir que ambos fueron amigos anteriormente, cuando ella era pareja de otro hombre (Y) que también formaba parte del grupo de amigos -más bien del triángulo-: "ellos dos cogidos de las manos... yo ayudándolos a desembarcar", "ellos allá, en la pista de baile, abrazados, muy juntos [...] acariciándose las mejillas, rozándose los labios”. En el presente de la narración, X es abandonado por Elisa, a causa del dolor que le produce el recuerdo del antiguo amor. ${ }^{12}$

$\mathrm{X}$ construye su discurso narrativo sobre la base de las impresiones que tiene del momento que vive mientras tiene lugar la despedida, las imágenes de su último encuentro con Elisa que le vienen a la mente y los recuerdos de ese pasado en que los tres estaban juntos. Por tanto, la historia es construida a partir de la convivencia del pasado y el presente en el pensamiento de X.

\subsubsection{Análisis sintáctico}

Después de dividir el texto en CO (184) y oraciones (345) podremos obtener la caracterización sintáctica del mismo (v. Anexo 1), de la que es posible extraer la siguiente información:

a) Tipo oracional

El 85,51\% de las oraciones es bimembre (295 oraciones) y el 14,49\% es unimembre (50 oraciones). Aunque el elevado porcentaje de oraciones bimembres es algo común, lo 
significativo en el texto es la variedad de estructuras que presentan las unimembres, pues son utilizadas con un determinado objetivo.

\section{a.1. Oraciones unimembres impersonales: 8 (16\%)}

Se usan para dar las circunstancias temporales: desde cuándo tiene lugar la relación de Elisa y X ("hace dos años"), hace cuánto tiempo "desapareció" Y ("hace once, doce años"), en qué momento tiene lugar la historia narrada ("Es sábado por la tarde") y cuál es la situación meteorológica ("Llueve con ganas”). Esta última es la más importante, pues la mención de la lluvia es la que nos permitirá definir en algunas ocasiones cuándo se habla del presente y cuándo del pasado:

[Pasado]: ellos dos cogidos de las manos... yo ayudándolos a desembarcar. Entramos en el bar, un muelle techado y sin paredes que se proyectaba hacia el mar. Un vaso de vino tinto y dos cervezas; de parte de ellos, abrazos, besos en las manos y las mejillas, en las bocas, sonrisas, susurros a los oídos, como hacen los amantes que no tienen pudor al mostrar en público su amor, con la necesidad de que todos sepan que es precisamente de amor de lo que se trata.

[Presente]: Llueve en la terraza del apartamento en que vivo. Llueve con ganas, es una lluvia constante, persistente [...]. La lluvia que cae y el ron que bebemos sorbo a sorbo. Elisa se levanta, va a la puerta ventana y recuesta la cabeza en las persianas sin que le importen las gotas de lluvia que se cuelan y la salpican.

Otra estructura impersonal empleada es la de la frase verbal con la estructura haber que + infinitivo: "Nos quedan el resto del sábado y parte del domingo para decirnos lo que haya que decir", "La cerveza se acaba, hay que pedir más". Por su campo semántico, esta frase verbal le da un carácter de obligatoriedad a la acción, pero al estar conjugada en tercera persona del singular indetermina al sujeto: la acción es de obligatorio cumplimiento para cualquiera de los personajes presentes en el contexto de enunciación de la frase verbal.

\section{a.2. Vocativo: 1 oración $(0,28 \%)$}

El único vocativo que aparece (“¿Qué miras, Elisa?”) tiene en esta ocasión un uso que contrasta con el habitual: el vocativo corresponde a la función apelativa del lenguaje, se emplea para llamar la atención del oyente, pero aquí no se llama la atención de ninguna persona. Acompaña a una pregunta que $\mathrm{X}$ formula en su mente y no exterioriza; entonces, no espera una respuesta ni una reacción por parte del oyente, pues él es al mismo tiempo hablante y oyente.

Como lectores, este vocativo nos sirve para saber que el objeto del interés de X es Elisa, él quiere saber qué piensa ella: "qué ocurre este sábado, porque algo diferente ocurre, algo que todavía no he podido descubrir... algo dentro de su cabeza rubia”. Este interés de X se refleja también en el hecho de que el único nombre que menciona en todo el texto es el de Elisa: en 11 ocasiones, incluyendo el título. De estas 11 apariciones, en las 10 oraciones que corresponden a su presencia funciona como núcleo, ${ }^{13}$ mientras que en la única oración que corresponde a su partida ya no es núcleo, sino objeto directo: "En la terraza, mojándome, la veo alejarse bajo la lluvia a ella, a mi Elisa”. Además, aparece no solo al final de la oración, sino 
también al final del texto, en una aposición, como si fuera una información más, algo que se ha añadido, y a la vez le da un carácter circular al cuento: comienza con el sustantivo Elisa en el título y termina con él. Podemos entonces decir que aquí se resume el texto: todo gira alrededor de Elisa, a la obsesión de X por ella.

\section{a.3. Nominales: 41 oraciones $(82 \%)$}

Las oraciones unimembres más usadas son las nominales. Su elevada frecuencia tiene como objetivo anular la sucesión temporal para dar lugar a la permanencia en el tiempo de las imágenes que se proyectan en la mente de $\mathrm{X}$ y son presentadas en el mismo momento de su producción. Veamos un ejemplo:

Risas y voces, luces rojas y amarillas, dedos que sujetan cigarrillos o tabacos, dientes perfectos o cariados, pechos enormes de matronas, gastados, ajados, guayaberas y anillos de oro y medallas colgando en los pechos de chulos trasnochados.

Se produce una aglutinación de sintagmas nominales - y al mismo tiempo de palabras - como forma de simular la dinámica del movimiento de los pensamientos de $\mathrm{X}$, en una larga cadena de asociación de ideas.

b) Categorías gramaticales que funcionan como núcleo predicativo

La tabla 1 nos permite comprobar que, como es común al uso de nuestra lengua, la categoría gramatical más empleada en el texto como núcleo predicativo es el verbo.

\begin{tabular}{|c|c|c|c|c|c|c|c|c|}
\hline & Verbo & Sustantivo & Adjetivo & Infinitivo & Gerundio & Participio & Pronombre & Adverbio \\
\hline Cantidad & 237 & 36 & 4 & 22 & 25 & 8 & 10 & 3 \\
\hline$\%$ & $68,70 \%$ & $10,43 \%$ & $1,16 \%$ & $6,38 \%$ & $7,25 \%$ & $2,32 \%$ & $2,90 \%$ & $0,87 \%$ \\
\hline
\end{tabular}

Tabla 1. Estadística de categorías gramaticales que funcionan como núcleo en "Elisa”

Para los propósitos del presente estudio, lo más destacable en el uso de los verbos es su comportamiento en cuanto a su omisión o no. De los 237 verbos, hay 21 omitidos y, además, entre esos verbos, hay 32 frases verbales de las cuales 4 tienen un elemento omitido (para un total de 25 omisiones, 10,55\%), como en los ejemplos: "Volvimos a estar, [volvimos] a fundirnos como la primera vez hace ya dos años", "cuando aún podíamos buscar en el mar incógnitas y la risa en las márgenes del mar de Regla, [podíamos] aspirar sus olores a mariscos, algas podridas, petróleo; [podíamos] improvisar aquellas palabras que nos decíamos por el sencillo placer de creernos algo poetas".

Otra vez la explicación es la rapidez del fluir del pensamiento o la intención de imitar estructuras propias del diálogo. El hablante no tiene tiempo de ordenar sus ideas ni de organizar sintácticamente sus palabras, por lo que en ocasiones se eliminan elementos. ${ }^{14}$ Pero ello no impide la comprensión del texto, pues estos han sido mencionados anteriormente, por lo que pueden ser inferidos. 
El sustantivo es la categoría que sigue en frecuencia al verbo (10,43\%). En este caso, 35 de las 36 oraciones que tienen como núcleo predicativo un sustantivo $(97,22 \%)$ son oraciones unimembres nominales (cf. supra). Solo hay una oración atributiva: "hoy es el día aquel en que lo vimos por última vez en el Parque Central”. La presencia de esta única oración atributiva con un sustantivo como núcleo predicativo llama la atención ya que corresponde con la enunciación del desencadenante de la separación de Elisa y X: el aniversario (?) de la "desaparición" de Y.

El gerundio es la forma no personal del verbo más empleada, con una frecuencia de aparición de 7,25\%. Esta categoría gramatical se usa con el propósito de dar al lector la sensación de estar asistiendo al desarrollo de la acción. Así se acentúa el matiz de perdurabilidad y, por tanto, la imparabilidad del pensamiento, que está en constante actividad. Aunque esté describiendo una circunstancia del pasado, como en "la que probaba mi cerveza y olía su vino, sonriente y transmitiendo con sus ojos misterios ocultos", expresa una acción no concluida; nos describe ese pasado en su duración.

c) Tipos estructurales de conjuntos oracionales (TECO) presentes

A partir de la relación de TECO (v. Anexo 2) podemos determinar otras características del texto como las siguientes:

\section{c.1. Frecuencia de oraciones intercaladas e interrumpidas}

Hay 15 oraciones interrumpidas (4,35\%) y 17 intercaladas (4,97\%) que se distribuyen en: 9 oraciones interrumpidas con 1 oración intercalada (60\%), 1 oración interrumpida con 2 oraciones intercaladas $(6,67 \%), 1$ oración interrumpida con 3 oraciones intercaladas $(6,67 \%)$ y 2 oraciones interrumpidas 2 veces con 1 oración intercalada en cada ocasión (13,33\%). El uso de tantas oraciones interrumpidas e intercaladas se justifica por el estilo entrecortado empleado en la narración debido a la rapidez con que fluye el pensamiento de X. Estas interrupciones en la coherencia de la narración se conectan con las repentinas interrupciones que se producen en la mente de $\mathrm{X}$ mediante procesos asociativos que van encadenando un pensamiento a otro. Veamos algunos ejemplos:

${ }^{1}$ Me ha besado ${ }^{2}$ como ella besa ${ }^{3}$ cuando nos vemos después de días sin ${ }^{4}$ encontrarnos, ${ }^{5}$ con ansias insatisfechas.

X ha dejado para el final de la oración lo que más importa desde la posición del lector: cómo Elisa lo ha besado. Desde su posición de personaje, lo más importante para X es recordar cuándo ella lo besa de esa manera; por eso deja para el final la caracterización modal de la acción besar. 


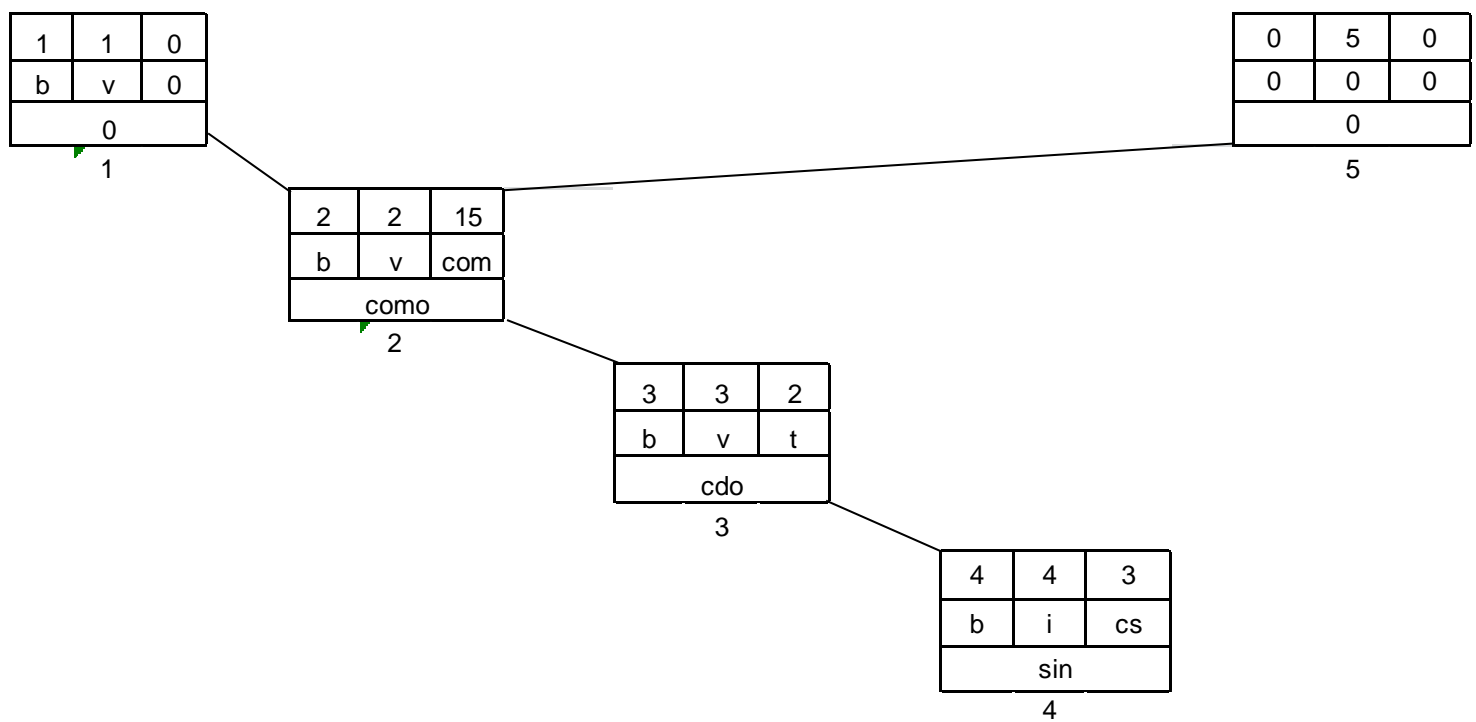

Como se aprecia en la estructura básica compleja del CO, el lector tiene que esperar al quinto segmento oracional para saber cómo Elisa besa a X. Y en el intermedio se le va dando cierta información que, a los efectos de comprensión de la historia contada, no son de interés.

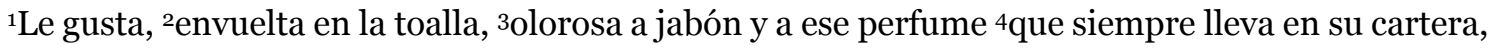
5recostarse sobre mi cama a ${ }^{6}$ esperar por el cigarrillo 7 que enciendo y ${ }^{8}$ pongo en sus labios.

En este ejemplo, lo que primero le viene a la mente a $\mathrm{X}$ es el modo en que a Elisa le gusta recostarse sobre la cama; más que lo que le gusta hacer, lo que recuerda $\mathrm{X}$ es la circunstancia modal que rodea a la acción.

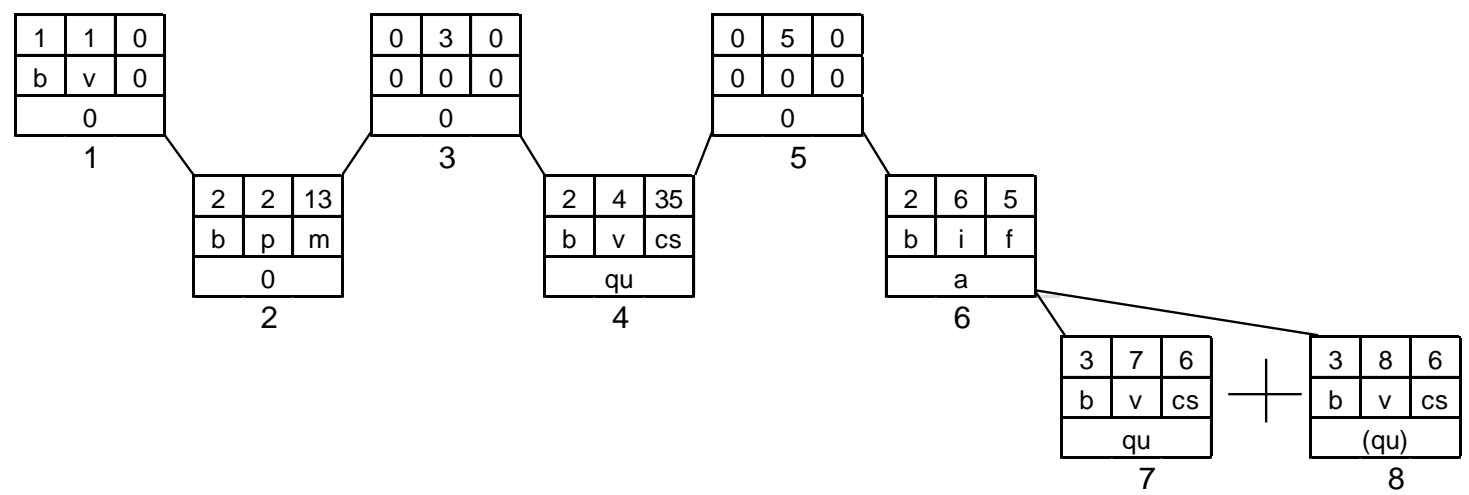

Aquí también el lector tiene que esperar al quinto segmento oracional para completar la información. Así pues, al usar oraciones interrumpidas con una o varias oraciones intercaladas se marca la interrupción de la narración de los hechos para introducir una secuencia descriptiva o alguna reflexión sobre estos. 
Otro elemento que ilustra el estilo entrecortado es el uso de oraciones yuxtapuestas que complementan la información dada por X en su narración, constituyendo series enumerativas con las que el ritmo se hace más acelerado, sobre todo cuando son oraciones unimembres nominales o sintagmas: "solo atinaba a responder con la misma intensidad al alucinante y progresivo acto en que nuestros cuerpos se entrelazaban agitándose, transpirando, suspirando con fuerza, a galope en busca del clímax sosegador", "Entramos en el bar, un muelle techado y sin paredes que se proyectaba hacia el mar. Un vaso de vino tinto y dos cervezas; de parte de ellos, abrazos, besos en las manos y las mejillas, en las bocas sonrisas, susurros a los oídos”. En ocasiones, también empleando varios elementos que cumplen una misma función como complementos verbales que modifican determinadas acciones. $\mathrm{X}$ va añadiendo las ideas a medida que vienen a su mente. Por ejemplo:

\section{[...] la veo alejarse a ella, a mi Elisa.}

Sale del cuarto, de mi cuarto, de nuestro cuarto. ${ }^{15}$

[...] hizo algún comentario acerca de la calle, la lluvia, la gente que se guarecía en las paradas de los ómnibus, de la tarde.

En el último ejemplo, hay 4 construcciones que cumplen la función de complemento circunstancial de asunto, lo que va alargando la oración principal y la va haciendo más compleja. Lo mismo ocurre en el $\mathrm{CO} 49$, en el que hay 6 oraciones subordinadas en función de complemento circunstancial de tiempo y, a su vez, cada una de ellas tiene otra(s) oración(es) subordinada(s), y así van haciendo más y más larga la cadena de subordinación a medida que surgen más recuerdos en la mente de $\mathrm{X}$.

93la mirada fija en el rectangular espejo ${ }^{94}$ que cuelga de la pared izquierda del cuarto, ${ }^{95} \mathrm{con}$ los mismos ojos de tantos años atrás, verdes tan verdes como el mar ${ }^{96}$ que cruzábamos en la lancha de Regla, ${ }^{97}$ cuando aún no sabíamos que ${ }^{98}$ alguna vez nos amaríamos, ${ }^{99}$ cuando todavía éramos tan jóvenes que ${ }^{100}$ podíamos aparentar ${ }^{101}$ no serlo, ${ }^{102}$ cuando la risa de la despreocupación nos impulsaba a ${ }^{103}$ ser insolentes con nosotros mismos y con los demás, ${ }^{104}$ cuando pensábamos en el tú después del yo y ${ }^{105}$ muy pocas veces en el nosotros, ${ }^{106}$ cuando aún podíamos buscar en el mar incógnitas y la risa en las márgenes del mar de Regla, ${ }^{107}$ aspirar sus olores a mariscos, algas podridas, petróleo; ${ }^{108 i m p r o v i s a r ~ a q u e l l a s ~ p a l a b r a s ~}{ }^{109}$ que nos decíamos por el sencillo placer de ${ }^{110}$ creernos algo poetas: ${ }^{111 “}$ "Tu imagen se mece en el viento del mar de Regla y ${ }^{112}$ mientras tanto yo, con mis 19 años, te contemplo con ojos de esperanza”; 113“Lanzan tus ojos risas de una primavera burlada"

El extremo de este estilo entrecortado es lo que ocurre en el CO 176: "Tu imagen se mece en el viento del mar de Regla y mientras tanto yo...”. La oración subordinada introducida por el nexo mientras tanto tiene omitido su predicado: su significación no está completa. Hay una interrupción en lo que se está diciendo. Esta expresión ya fue enunciada anteriormente, por lo que a efectos de la lectura no es necesario que aparezca en su totalidad: su contenido puede sobrentenderse. Al no ser enunciada del todo, se usan los puntos suspensivos para expresar la continuidad de la acción y con ello del flujo de pensamiento, como también ocurre en los CO $54,66,92,111,122,144,145,153,172$ y 175. 
c.2. Frecuencia de niveles en que se encuentran las oraciones subordinadas

\begin{tabular}{|c|c|c|c|c|c|c|c|c|c|c|c|c|c|c|}
\hline \multirow{2}{*}{$\begin{array}{l}\text { NIVEL } \\
\text { ORAC. }\end{array}$} & \multicolumn{2}{|c|}{1} & \multicolumn{2}{|c|}{2} & \multicolumn{2}{|c|}{3} & \multicolumn{2}{|c|}{4} & \multicolumn{2}{|c|}{5} & \multicolumn{2}{|c|}{6} & \multicolumn{2}{|c|}{7} \\
\hline & Cant. & $\%$ & Cant. & $\%$ & Cant. & $\%$ & Cant. & $\%$ & Cant. & $\%$ & Cant. & $\%$ & Cant. & $\%$ \\
\hline & 185 & 53,62 & 92 & 26,67 & 42 & 12,17 & 16 & 4,64 & 7 & 2,03 & 2 & 0,58 & 1 & 0,29 \\
\hline \multicolumn{3}{|c|}{$\begin{array}{c}\text { TOTAL ORACIONES } \\
\text { SUBORDINADAS }\end{array}$} & \multicolumn{12}{|c|}{160} \\
\hline
\end{tabular}

Tabla 2. Estadística de niveles oracionales en "Elisa”

Como podemos ver en la tabla 2, hay un gran encadenamiento de oraciones subordinadas, dado por la cantidad de niveles de subordinación que se completan en el texto: llega hasta un séptimo nivel, ${ }^{16}$ lo que refleja la actividad del pensamiento, en la que se encadenan muchas oraciones que van de un pensamiento a otro, como ocurre en el $\mathrm{CO} 49,{ }^{17}$ en el que se van sucediendo circunstancias temporales que completan la información de la oración 104: "que [el mar] cruzábamos en la lancha de Regla", una subordinada que complementa al sustantivo el mar, el cual forma parte de otra subordinada que modifica a la oración principal (101), interrumpida a su vez por otra subordinada en función de complemento de sustantivo: "93la mirada fija en el rectangular espejo ${ }^{94}$ que cuelga de la pared izquierda del cuarto, ${ }^{95}$ con los mismos ojos de tantos años atrás, verdes tan verdes como el mar ${ }^{96}$ que cruzábamos en la lancha de Regla”. Al mismo tiempo, las circunstancias temporales de cruzar el mar son modificadas por varias oraciones subordinadas con diversas funciones, haciendo más largo el $\mathrm{CO}$, que comenzó como una oración unimembre nominal: "la mirada fija en el rectangular espejo que cuelga de la pared izquierda del cuarto".

\section{c.3. Frecuencia de estilo directo}

De los 184 CO del texto, hay 8 (4,35\%) con oraciones en estilo directo; de ellos, 7 (3,80 \%) representan la conversación que sobre su relación $\mathrm{X}$ tuvo con Elisa el día de la despedida y que él recuerda una y otra vez. Por eso se repite la misma estructura (-110), así como la negación de Elisa. Más que para marcar la presencia de un diálogo, el estilo directo se usa para destacar la parte del diálogo que ha quedado en la mente de X; más que lo que hablan los dos, es lo que él recuerda, de ahí la ausencia de verbos de lengua y de una intervención de X para introducir los parlamentos.

Hasta aquí tenemos una breve y rápida caracterización del cuento "Elisa". Ahora trataremos de compararlo con el cuento "Miedo" con el propósito de definir cuáles son las características que definen en "Elisa" el uso de la técnica del flujo de conciencia.

\subsection{COMPARACIÓN CON "MIEDO"}

A grandes rasgos, podemos decir que en "Miedo" asistimos a la narración, por parte de un hombre, de los acontecimientos de su niñez que lo hicieron ser lo que es ahora. Este narradorpersonaje ${ }^{18}$ nos describe cada uno de los miedos que acompañaron a su niñez: miedo a dormir solo (por temer a la oscuridad y a los fantasmas y muertos que parecía ver) y miedo al vecino "desagradable y vulgar”. 
Contrario a lo que sucede en "Elisa", el discurso narrativo es construido siguiendo una linealidad temporal: desde un presente muy alejado en el tiempo del pasado que recuerda, $\mathrm{Z}$ narra los hechos de su niñez hasta llegar a la adultez. Por esta razón la sintaxis es mucho más organizada y coherente; ya no se trata de un fluir del pensamiento más espontáneo, que enlaza presente y pasado una y otra vez. El objetivo aquí es buscar las causas de su miedo, no reflexionar sobre él; es como una búsqueda de respuestas a su comportamiento actual a través de la historia pasada.

En el otro extremo, la estructura narrativa de "Elisa” es más caótica, pues está construida sobre la base de las imágenes y recuerdos que se suceden en la mente del narradorprotagonista y en lo que este siente hacia ellos y hacia el presente. Veamos cómo contrasta esto sintácticamente con la caracterización de "Miedo". Igual que hicimos con el cuento anterior, debemos ver la división del texto en CO (182) y oraciones (296) para obtener la caracterización sintáctica (v. Anexo 3). De aquí podremos extraer las siguientes características:

\section{a) Tipo oracional 19}

Como sucede en "Elisa”, las oraciones bimembres son las de más frecuente uso en "Miedo" (véase tabla 3); lo destacable es la disminución de oraciones unimembres. ${ }^{20}$

\begin{tabular}{|c|c|c|c|c|}
\hline \multirow{2}{*}{ TEXTO } & \multicolumn{2}{|c|}{ UNIMEMBRES } & \multicolumn{2}{c|}{ BIMEMBRES } \\
\cline { 2 - 5 } & Cantidad & $\%$ & Cantidad & $\%$ \\
\hline "ELISA" & 50 & $14,49 \%$ & 295 & $85,51 \%$ \\
\hline "MIEDO" & 20 & $6,76 \%$ & 276 & $93,24 \%$ \\
\hline
\end{tabular}

Tabla 3. Comparación estadística de las oraciones unimembres y bimembres de ambos cuentos

En el texto analizado ahora, las más usadas son las impersonales: 10 (50\%), en primer lugar, las del verbo haber: 6 (30\%), en la descripción del ambiente que rodeaba a $\mathrm{Z}$ en su niñez. $\mathrm{Al}$ estar conjugado en copretérito de indicativo, el verbo expresa una existencia pasada cuyo principio y fin no nos interesa. Algo diferente a lo que ocurre en "Elisa": las oraciones unimembres más usadas son las nominales, para mantener la anulación temporal; imágenes del pasado evocadas en el presente y enunciadas tal y como vienen a la mente del sujeto enunciador. En "Miedo", además, llama la atención el uso de las interjecciones (2), incluidas en $\mathrm{CO}$ que corresponden a parlamentos de un diálogo, y mantienen así su naturaleza semántica: expresión de sentimientos, actitudes y sensaciones del hablante.

b) Categorías gramaticales que funcionan como núcleo predicativo

La tabla 4 nos muestra que, en ambos textos, la categoría gramatical más usada como núcleo predicativo es el verbo conjugado. 


\begin{tabular}{|l|c|c|c|c|}
\hline \multirow{2}{*}{ CATEGORÍA } & \multicolumn{2}{|c|}{ "ELISA" } & \multicolumn{2}{c|}{ "MIEDO" } \\
\cline { 2 - 5 } & Cantidad & $\%$ & Cantidad & $\%$ \\
\hline verbo & 237 & $68,70 \%$ & 215 & $72,64 \%$ \\
\hline sustantivo & 36 & $10,43 \%$ & 15 & $5,07 \%$ \\
\hline adjetivo & 4 & $1,16 \%$ & 16 & $5,41 \%$ \\
\hline infinitivo & 22 & $6,38 \%$ & 35 & $11,82 \%$ \\
\hline gerundio & 25 & $7,25 \%$ & 3 & $1,01 \%$ \\
\hline participio & 8 & $2,32 \%$ & 3 & $1,01 \%$ \\
\hline pronombre & 10 & $2,90 \%$ & 2 & $0,68 \%$ \\
\hline adverbio & 3 & $0,87 \%$ & 3 & $1,01 \%$ \\
\hline oración & - & - & 3 & $1,01 \%$ \\
\hline interjección & - & - & 1 & $0,34 \%$ \\
\hline
\end{tabular}

Tabla 4. Comparación estadística de las categorías gramaticales que funcionan como núcleo predicativo en ambos cuentos

Ya vimos que en "Elisa" hay 25 omisiones verbales (10,55\%); en "Miedo" hay 13 omisiones verbales y 1 omisión de elementos de frases verbales: 14 en total (6,51\%). "Elisa" casi duplica en omisiones verbales a "Miedo". La explicación puede ser la intención que tiene el autor de imitar el espontáneo fluir del pensamiento. Es significativa la baja frecuencia de aparición que posee el gerundio con respecto a "Elisa", donde ya vimos que tiene una intención de marcar la continuidad de la acción expresada por esta categoría verbal y también el continuo fluir de las ideas en el pensamiento de $\mathrm{X}$.

Los adjetivos también son muy frecuentes en "Miedo". En el texto se realizan muchas descripciones y, por tanto, se necesitan muchos adjetivos, como ocurre en el CO 9: "Era feo, rechoncho, de baja estatura, peludo y hosco como un oso gruñón".

c) Tipos estructurales de conjuntos oracionales (TECO) presentes

En el Anexo 4 están relacionados los TECO de "Miedo", los que nos permiten comparar este cuento con "Elisa" teniendo en cuenta los siguientes aspectos:

\section{c.1. Frecuencia de oraciones intercaladas e interrumpidas}

En "Miedo" hay 6 oraciones interrumpidas (2,03\%), distribuidas en: 5 oraciones interrumpidas con 1 oración intercalada y 1 interrumpida con $1 \mathrm{CO}$ intercalado. Con respecto a "Elisa", la frecuencia de oraciones interrumpidas e intercaladas es muy baja, precisamente porque su objetivo no es el de mostrar las constantes interrupciones de un proceso de pensamiento; incluso las interrupciones son muy cortas. Aunque sí está el estilo entrecortado: hay ocasiones en las que la oración es completada después del punto y seguido, como para ir añadiendo informaciones que especifiquen lo dicho anteriormente. Por ejemplo: "Cerca había un muelle de madera donde mis amigos y yo pescábamos todos los días. Pequeños peces y cangrejos" y "Supongo que no tenía nada contra mí, sino que simplemente querían estar solos. Para tener sexo". Al poner el complemento independiente después del punto, se hace una 
especie de nota aclaratoria en la que se destaca el complemento añadido; es lo que más queda en el lector después de recibir toda la información dada.

\section{c.2. Frecuencia de niveles en que se encuentran las oraciones}

Como puede apreciarse en la tabla 5, "Miedo" tiene menor número de niveles de subordinación que "Elisa", lo que hace a este primer texto menos profundo sintácticamente y de mejor comprensión por parte del lector, pues los límites entre una oración y otra no están tan difusos como en "Elisa".

\begin{tabular}{|c|c|c|c|c|}
\hline NIVEL ORACIONAL & \multicolumn{2}{|c|}{ "ELISA" } & \multicolumn{2}{c|}{ "MIEDO" } \\
\hline & Cantidad & $\%$ & Cantidad & $\%$ \\
\hline 1 & 185 & 53,62 & 188 & 63,51 \\
\hline 2 & 92 & 26,67 & 90 & 30,41 \\
\hline 3 & 42 & 12,17 & 14 & 4,73 \\
\hline 4 & 16 & 4,64 & 4 & 1,35 \\
\hline 5 & 7 & 2,03 & - & - \\
\hline 6 & 2 & 0,58 & - & - \\
\hline 7 & 1 & 0,29 & - & - \\
\hline $\begin{array}{c}\text { TOTAL DE ORACIONES } \\
\text { SUBORDINADAS }\end{array}$ & \multicolumn{2}{|c}{$161^{21}$} & & $114^{22}$ \\
\hline
\end{tabular}

Tabla 5. Estadística niveles oracionales de ambos cuentos

Aunque entre ambos textos el número de $\mathrm{CO}$ complejos tiene una pequeña diferencia de cinco ("Elisa" tiene 171 y "Miedo" 166), ambos se distinguen en cuanto al nivel de complejidad: en "Elisa" es mayor por necesidades del contenido de la narración. Sintácticamente se refleja el sentir del narrador-personaje, la oscuridad de su pensamiento, construido sobre ideas e imágenes que se suceden en un movimiento constante y desordenado, como se muestra en el CO 49, ya analizado anteriormente: en esta ocasión solo nos valdremos del código numérico que lo identifica, el cual nos permite notar la complejidad de su estructura a partir de los muchos niveles de subordinación y las interrupciones en los $\mathrm{CO}$, así como el alto número de oraciones que lo componen: 1102213030243354465374487598 3A4 4BA 3C4 3D4 3E4 3F4 3G4 4HG 5IH :3JG 4KJ 3LG. Por otro lado, si volvemos a los anexos 2 y 4, podremos comprobar también que en "Elisa" hay más variedad de estructuras oracionales, lo que muestra una mayor elaboración en la sintaxis. A esta complejidad sintáctica hay que agregar también que "Elisa" está construido con más palabras (1703 frente a las 1509 de "Miedo") y tiene un mayor promedio de palabras por CO (9,26 frente a 8,29 en "Miedo") y de oraciones por CO complejo (4,86 frente a 4,48 en "Miedo"), lo que le da mayor volumen a las oraciones y con ellas a los CO.

Por otro lado, la complejidad de "Elisa" con respecto a "Miedo" se puede comprobar con la variedad de estructuras oracionales. Si volvemos a los anexos 2 y 4, podemos ver que ambos cuentos solo tienen en común $8 \mathrm{CO}$, que corresponden a las estructuras más simples (siendo la más frecuente, como es lógico, la oración simple), en dos de las cuales solo se llega a un tercer nivel de subordinación, y el máximo de oraciones constituyentes es de 4, en un CO. 


\section{c.3. Frecuencia de estilo directo}

El uso del estilo directo en "Miedo" es menos frecuente que en "Elisa": solo se usa en 6 CO $(3,3 \%)$ frente a los $8(4,35 \%)$ del otro cuento, aunque este número no es tan relevante si tenemos en cuenta que "Miedo" tiene $2 \mathrm{CO}$ y 49 oraciones menos. La diferencia entre ambos textos está en que en este último cuento el estilo directo se emplea para destacar la presencia de un diálogo entre los personajes (en "Elisa" se utiliza como cita de algo que dijo un personaje), tal como ocurre en cualquier narración, y solo en $3 \mathrm{CO}$ no hay presente ningún verbo de lengua para introducir los parlamentos, ${ }^{23}$ pero sí están identificados gráficamente con el uso de la pleca. Es tanto el interés por marcar las conversaciones, que se usan elementos propios del coloquio como las interjecciones, la partícula bueno como conector discursivo - da inicio al diálogo - y de algo característico de la sintaxis del lenguaje oral: la omisión de los verbos en las preguntas y la omisión de la oración principal en las respuestas, como en los CO 132 (“¿Por qué [no te gusta dormir solo]?”) y 133 (“[No me gusta dormir solo] Porque me da miedo"). ${ }^{24}$

Ya que hablamos de conectores discursivos, es importante destacar el uso de estos para enlazar CO. Mientras en "Miedo" es mayor la presencia de relaciones de coordinación entre CO (41, para un 22,65\%) que en "Elisa" (29, para un 15,85\%), en el segundo es mayor la presencia de relaciones de yuxtaposición (154, para un 84,15\%, frente a 140 en "Miedo", para un 77,35 \%). Ello muestra una mayor coherencia lógica entre los $\mathrm{CO}$ de "Miedo" frente al alto número de series enumerativas de "Elisa", por medio de la yuxtaposición de CO y oraciones, con lo que se transmite al lector la desesperación y el ahogo emocional que experimenta el narrador-protagonista.

Por último, habría que destacar el uso de las oraciones subordinadas y sus nexos. Veamos las tablas 6 y 7 .

\begin{tabular}{|c|c|c|c|c|}
\hline \multirow{2}{*}{ FUNCIÓN } & \multicolumn{2}{|c|}{ "ELISA } & \multicolumn{2}{c|}{ "MIEDO" } \\
\cline { 2 - 5 } & Cantidad & $\%$ & Cantidad & $\%$ \\
\hline complemento de sustantivo & 38 & 23,60 & 23 & 20,18 \\
\hline complemento de pronombre & 12 & 7,45 & 1 & 0,88 \\
\hline complemento de adjetivo & - & - & 1 & 0,88 \\
\hline sujeto & 5 & 3,11 & 5 & 4,39 \\
\hline objeto directo & 30 & 18,63 & 33 & 28,95 \\
\hline atributo & - & - & 1 & 0,88 \\
\hline complemento circunstancial & 3 & 1,86 & - & - \\
\hline c.c. modo & 29 & 18,01 & 2 & 1,75 \\
\hline c.c. tiempo & 27 & 16,77 & 4 & 3,51 \\
\hline c.c. finalidad & 3 & 1,86 & 13 & 11,40 \\
\hline c.c. comparación & 9 & 5,59 & 8 & 7,02 \\
\hline c.c. consecuencia & 1 & 0,62 & 4 & 3,51 \\
\hline c.c. causa & 3 & 1,86 & 11 & 9,65 \\
\hline c.c. concesión & - & - & 1 & 0,88 \\
\hline c. régimen & 1 & 0,62 & 1 & 0,88 \\
\hline c.c. condición & - & - & 6 & 5,26 \\
\hline
\end{tabular}

Tabla 6. Función de la oración subordinada en ambos cuentos 


\begin{tabular}{|c|c|c|c|c|}
\hline \multirow[b]{2}{*}{ NEXO } & \multicolumn{2}{|c|}{ "ELISA" } & \multicolumn{2}{|c|}{ "MIEDO" } \\
\hline & Cantidad & $\%$ & Cantidad & $\%$ \\
\hline 0 & 37 & 22,13 & 24 & 21,05 \\
\hline $\mathrm{a}$ & 6 & 3,75 & 5 & 9,39 \\
\hline al & 4 & 2,5 & - & - \\
\hline antes-de-q & - & - & 2 & 1,75 \\
\hline apenas & - & - & 1 & 0,88 \\
\hline así-q & - & - & 2 & 1,75 \\
\hline aunq & - & - & 1 & 0,88 \\
\hline $\mathrm{cdo}^{25}$ & 11 & 6,88 & - & - \\
\hline $\operatorname{com}^{26}$ & 8 & 5 & 8 & 7,02 \\
\hline como $^{27}$ & 2 & 1,25 & - & - \\
\hline con & - & - & 1 & 0,88 \\
\hline $\mathrm{dd}^{28}$ & 1 & 0,63 & 4 & 3,51 \\
\hline de & 4 & 2,5 & 5 & 4,39 \\
\hline de-q & 1 & 0,63 & 1 & 0,88 \\
\hline desde & 1 & 0,63 & - & - \\
\hline desde-q & 2 & 1,25 & - & - \\
\hline hasta & - & - & 1 & 0,88 \\
\hline igual-q & 1 & 0,63 & - & - \\
\hline mtras & 5 & 3,13 & - & - \\
\hline mtras-tto & 2 & 1,25 & - & - \\
\hline para & 1 & 0,63 & 10 & 8,77 \\
\hline por & - & - & 1 & 0,88 \\
\hline por-que 29 & - & - & 1 & 0,88 \\
\hline $\mathrm{pq}^{30}$ & 4 & 2,5 & 9 & 7,89 \\
\hline$q^{31}$ & 17 & 10,63 & 18 & 15,79 \\
\hline $\mathrm{qu}^{32}$ & 40 & 25 & 12 & 10,53 \\
\hline que 33 & 5 & 3,13 & 1 & 0,88 \\
\hline qui34 & 2 & 1,25 & - & - \\
\hline si & 1 & 0,63 & 6 & 5,26 \\
\hline $\sin$ & 3 & 1,88 & - & - \\
\hline $\sin 0-q$ & - & - & 1 & 0,88 \\
\hline $\sin -q$ & 1 & 0,63 & - & - \\
\hline tal-y-com & 1 & 0,63 & - & - \\
\hline
\end{tabular}

Tabla 7. Nexos subordinantes de ambos cuentos

Después de analizar las estadísticas que nos presentan ambas tablas, vemos que, a pesar de que en "Miedo" hay un porcentaje menor de oraciones subordinadas y de niveles de subordinación, es más variado el uso de estas oraciones: hay diversidad en cuanto a la función desempeñada por sus oraciones subordinadas. Incluso, es más variado el uso de los nexos subordinantes, lo que nos permite considerar una diferencia con "Elisa": como en "Miedo" la enunciación no responde a un flujo espontáneo de pensamiento, alejado de la lógica sintáctica, el sujeto enunciador se permite hacer un uso menos reiterativo de estructuras subordinadas y, para no incurrir en repeticiones, busca nexos diferentes para introducir oraciones con la misma función sintáctica. 
En la confrontación de las estadísticas de las funciones desempeñadas por las oraciones subordinadas en ambos textos vemos que la mayor diferencia en cuanto a frecuencia de uso está en las oraciones con función de complemento circunstancial de modo y de tiempo. En "Elisa" son más frecuentes porque, en los $\mathrm{CO}$ en los que aparecen, $\mathrm{X}$ da importancia a las circunstancias expresadas por estas oraciones subordinadas, por lo que va enunciando una y otra vez oraciones temporales y modales. De ahí que los nexos más frecuentes sean los que habitualmente introducen oraciones de este tipo, así como el '0', que corresponde al uso de formas no personales del verbo.

Algo parecido sucede con las complementarias de sustantivo y pronombre, que son las más usadas para añadir información sobre el tema del que habla: caracteriza al objeto de su interés, y con esta caracterización brinda al lector cierta información relevante para él como personaje. Otra diferencia entre ambos textos es que en "Miedo" es mayor el uso de subordinadas causales, pues el protagonista narra en el cuento las causas de su comportamiento presente y las causas de sus miedos pasados.

\section{CONCLUSIONES}

Tras este análisis, queda demostrado cuán útil puede resultar el método propuesto por el profesor Rodríguez para la caracterización lingüística de textos, en este caso, para la identificación de determinados rasgos sintácticos que reflejan el contenido expresado en un cuento. A partir de los datos estadísticos obtenidos y la comparación de los dos cuentos analizados, podemos concluir que las características que definen el estilo del cuento "Elisa" en cuanto al empleo de la técnica narrativa del flujo de pensamiento son:

1) Elevado uso de oraciones unimembres nominales para dar lugar a la permanencia en el tiempo de las imágenes evocadas en la mente del protagonista.

2) Aglutinación de sintagmas nominales y palabras para simular el continuo movimiento de las ideas en la conciencia, así como un elevado promedio de palabras por $\mathrm{CO}$ y oraciones por $\mathrm{CO}$ complejo.

3) Frecuentes omisiones de verbos y partes de frases verbales, incluso del predicado en una oración.

4) Elevada frecuencia de uso de gerundios, con el propósito de dar al lector la sensación de constante actividad del pensamiento.

5) Frecuente uso de oraciones intercaladas e interrumpidas y, con ellas, de un estilo entrecortado en la narración.

6) Encadenamiento de muchas oraciones subordinadas.

7) Poco uso de conectores para enlazar CO.

Estas características sintácticas sirven de apoyo al contenido del cuento, donde se muestra la frustración y el dolor de X por la partida de Elisa. El texto representa un continuo fluir de imágenes y reflexiones, en el pensamiento de X, sobre los hechos que narra. Se producen constantes procesos asociativos que unen un pensamiento a otro, mediante el encadenamiento de un CO (u oración) a otro(a). A través del contenido y las estructuras sintácticas que emplea el autor para la construcción de su discurso, se logra acercar el lector al protagonista y sus 
sentimientos hacia lo que ocurre. La "oscuridad" y la complejidad sintáctica del texto corresponden a una “oscuridad” en X y a la compleja dinámica de su pensamiento.

\section{NOTAS}

1 Ya sea en su propia voz, en la voz de los personajes o en la del narrador.

2 "[...] los hechos de expresión del lenguaje organizado desde el punto de vista de su contenido afectivo, es decir, la expresión de los hechos de la sensibilidad mediante el lenguaje y la acción de los hechos del lenguaje sobre la sensibilidad". Todas las traducciones son mías.

3 “Cada característica del lenguaje es una cuestión de diseño, antes que de azar o descuido".

4 "Si quedan algunos críticos que aún dudan de la competencia de la lingüística para incluir el campo de la poética, yo personalmente creo que la falta de interés por la poética que muestran algunos lingüistas fanáticos ha sido confundida con una incapacidad de la ciencia lingüística en sí. [...] un lingüista que ignora la función poética del lenguaje y un estudioso de la literatura indiferente a las cuestiones relativas a la lengua y que no esté al corriente de los métodos lingüísticos son por igual anacronismos flagrantes".

5 El conocimiento de la propuesta de esta profesora no es resultado de la consulta bibliográfica, sino de las experiencias obtenidas en clases, así como de la consulta de las orientaciones metodológicas impartidas a profesores universitarios. Gran parte de los presupuestos teóricos elaborados por García Cortiñas fueron puestos en práctica en los manuales de estudio de Gramática Española empleados en la Facultad de Artes y Letras, a los cuales pertenecen las citas mencionadas aquí.

6 Definida como "toda estructura formada por un sujeto: de quién o de qué se habla, y un predicado: lo que se dice del sujeto" (Cueva, 1982, 48).

7 Definida como "la unidad intencional y atencional de sentido completo en sí misma, cuyo signo lingüístico es la cadencia". En ella "se comunica todo lo relativo a un mismo sujeto o todo lo que el hablante considere íntimamente relacionado". Su fin "se marca fonéticamente por la cadencia: una inflexión descendente, el tono normal del hablante baja; y en el lenguaje escrito se indica por un punto" (Cueva, 1982, 47).

8 Para las relaciones de coordinación disyuntiva y adversativa se emplean los símbolos $\sim_{y} \times$, respectivamente, y para la yuxtaposición la barra (/).

9 Para el listado completo de símbolos propuestos, cf. Rodríguez Suárez, 1989, 169-171.

10 Nótese que en el nuevo sistema no se marcan las relaciones de coordinación y yuxtaposición entre $\mathrm{CO}$, puesto que no es de interés para los objetivos propuestos.

11 Como no se menciona su nombre y constantemente se hará referencia a este narrador-personaje, para hacer más cómodo el proceso de escritura y evitar constantes repeticiones del término, a partir de este momento aparecerá referido como X.

12 O la vergüenza por estar con otro hombre. Nunca sabremos realmente cuál es la causa: solo sabemos que la despedida es desencadenada por el hecho de que ese último día en el que se encuentran es el mismo en que vieron por última vez a Y, "que desapareció hace once, doce años, que se lo tragó la tierra, que lo mataron" (¿aniversario?).

13 Ya sea del sujeto o de una oración unimembre nominal.

14 Llegando incluso a la omisión de todo el predicado de una oración, como veremos más adelante. 
15 En este caso, los añadidos muestran una gradación de lo general a lo particular que expresa la existencia de una relación entre $\mathrm{X}$ y Elisa a través del uso del artículo y de pronombres posesivos: va de el cuarto a nuestro cuarto. Al tiempo que constituyen una suerte de reformuladores de lo dicho, como una muestra de los pensamientos "obsesivo-repetitivos" de $\mathrm{X}$ con respecto a Elisa. Y, como en el primer ejemplo, adquieren valor enfático.

16 Esto hace que el texto tenga una gran profundidad sintáctica.

El que más oraciones tiene: 20 (el 5,80 \% de las oraciones del texto).

Aquí será referido como Z.

19 Como se pretende hacer un estudio comparativo para definir las características de un estilo determinado, la caracterización de "Miedo" se llevará a cabo a partir de los elementos que permitan realizar una comparación con "Elisa", con el fin de definir cuáles serían los que podrían contribuir a distinguir y definir el estilo del fluir del pensamiento en "Elisa".

Más allá del hecho de que el texto presente un menor número de $\mathrm{CO}$ y oraciones.

Según la suma de oraciones, en los niveles del 2 al 7 hay 160 oraciones subordinadas, pero realmente son 161, pues se cuentan también las oraciones en estilo directo, que aparecen marcadas en el primer nivel, por necesidades del método presentado, pues estilísticamente son consideradas oraciones independientes (Rodríguez Suárez, 1989 184-186), pero no sintácticamente. Es por eso por lo que, pese a que el código numérico las representa en el primer nivel oracional, en la casilla correspondiente al número de la oración a la que se subordinan no aparece un '0' como corresponde a las oraciones no subordinadas, y en la casilla de la función todas tienen puesta la de objeto directo.

22 Según la suma de oraciones, en los niveles 2, 3 y 4 hay 108 oraciones subordinadas, pero realmente son 114 (véase nota anterior).

Son los CO que corresponden a las respuestas de otros personajes. Los verbos de lengua están usados en los otros tres $\mathrm{CO}$, que son los que dan inicio a los diálogos.

24 De ahí que en la tabla de las características sintácticas de este texto el CO aparezca caracterizado con el código (-110) 22(1), en el que los paréntesis indican que la primera oración no aparece enunciada en el texto.

Cuando. Solo se aclaran los nexos cuyas abreviaturas pueden provocar confusión en la lectura. Para el listado completo de abreviaturas propuestas, véase Rodríguez Suárez (1989, 169-171).

Como.

Cómo.

Donde.

Por qué.

Porque.

Que (conjunción subordinante).

Que (pronombre relativo).

Qué (pronombre interrogativo o exclamativo).

Quien. 


\section{REFERENCIAS BIBLIOGRÁFICAS}

ALDAMA PANDO, I. 2017. "La literatura como intercambio verbal. Aplicación del modelo de las implicaturas conversacionales al análisis de un texto narrativo", Santiago, 143, pp. 359-374.

ALONSO, A. 1955. "Epístola a Alfonso Reyes sobre la estilística", en Alonso, A., Materia y forma en poesía. Madrid: Gredos, pp. 78-86.

ALONSO, D. 1950. Poesía española. Ensayo de métodos y límites estilísticos. Madrid: Gredos.

ARKINS, B. 1994. "Passionate syntax: style in the poetry of Yeats", en Finneran, R. (ed.), Yeats: an annual of critical and textual studies. Michigan: University of Michigan Press, pp. 3-26.

AUSTIN, J. L. 1976. How to do things with words. Oxford: Oxford University Press.

BAKER, W. E. 1967. Syntax in English poetry 1870-193o. Berkeley: University of California Press.

BALLY, C. 1905. Précis de stylistique. Ginebra: Eggiman.

BALLY, C. 1909 [1951]. Traité de Stylistique française (3. ${ }^{\mathrm{a}}$ ed.). París: Klincksieck.

BRUNEAU, C. 1970 [1951]. "La Stylistique", en Guiraud, P. y Kuentz, P., La Stylistique. Lectures.

París: Klincksieck, pp. 24-26.

BURUNAT, S. 1980. El monólogo interior como forma narrativa en la novela española (19401975). Madrid: José Porrúa Turanzas.

CALSAMIGLIA, H. y TUSÓN, A. 1999. Las cosas del decir. Manual de análisis del discurso. Barcelona: Ariel.

CAMPS, D. 2009. "Elisa", La Siempreviva, 7, pp. 10-13.

COOK, Guy. 1994. Discourse and literature. The interplay of form and mind. Oxford: Oxford University Press.

CRESSOT, M. 1969 [1947]. Le Style et ses techniques (6. ${ }^{\mathrm{a}}$ ed.). París: Klincksieck.

CUEVA, O. DE LA. 1982. Manual de gramática española (t. I). La Habana: Universidad de La Habana, Facultad de Artes y Letras.

DAVIE, D. 1966. Articulate energy: an enquiry into the syntax of English poetry. Londres: Routledge. DOLEŽEL, L. y BAILEY, R. W. 1969. Statistics and style. Nueva York: American Elsevier.

FOWLER, R. 1971. The languages of literature: some linguistic contributions to criticism. Londres: Routledge and Kegan Paul.

FOWLER, R. 1981. Literature as social discourse: the practice of linguistic criticism. Bloomington: Indiana University Press.

FOWLER, R. 1986. Linguistic criticism. Oxford: Oxford University Press.

FOWLER, R., HODGE, G., KRESS, G., y TREW, T. 1979. Lenguaje y control. México: Fondo de Cultura Económica.

GRICE, P. 1975. "Lógica y conversación”, en Valdés, L. M. (ed.), La búsqueda del significado. Madrid: Tecnos / Universidad de Murcia, pp. 511-530.

GUTIÉRREZ, P. J. 2009. “Miedo”, La Siempreviva, 5, pp. 52-54.

HODGE, R. y KRESS, G. 1979. Language as ideology. Londres: Routledge.

JAKOBSON, R. 1960. "Concluding statement: linguistics and poetics”, en Sebeok, T. A. (ed.), Style in language. Cambridge, Mass.: The MIT Press, pp. 350-377.

KEARNS, M. 1999. Rhetorical narratology. Lincoln: University of Nebraska Press.

LECERCLE, J. 1999. Interpretation as pragmatics. Londres: Macmillan.

LEECH, G. N. 1969. A linguistic guide to English poetry. Londres: Longman.

LEECH, G. N. 1976. "Literary criticism and linguistic description", Dutch Quarterly Review of Anglo-American Letters, 7, pp. 2-22.

MAROUZEAU, J. 1959. Précis de stylistique Française. París: Masson.

MILIC, L. T. 1967. A quantitative approach to the style of Jonathan Swift. La Haya: Mouton. 
OHMANN, R. 1971. "Speech acts and the definition of literature", Philosophy and Rhetoric, 4(1), pp. 1-19.

PRATT, M. L. 1977. Toward a speech act theory of literary discourse. Bloomington: Indiana University Press.

PRETORIUS, W. G. J. 1981. “A linguistic analysis of certain poems by T. S. Eliot”. Trabajo Fin de Máster. Potchefstroom: Potchefstroom University for Christian Higher Education.

RODRÍGUEZ SUÁREZ, L. E. 1989. "Sintaxis de la norma culta escrita en Cuba: cuento y artículo periodístico", en El español en Cuba. Anuario. La Habana: Facultad de Artes y Letras, Universidad de La Habana, pp. 163-190.

SEARLE, J. R. 1969. Speech acts. An essay in the philosophy of language. Cambridge: Cambridge University Press.

SELL, Roger D. 2000. Literature as communication. Amsterdam: John Benjamins.

SPERBER, D. y WILSON, D. 1986 [1994]. La relevancia. Comunicación y procesos cognitivos. Madrid: Visor.

SPITZER, L. 1931. "La interpretación lingüística de las obras literarias", en Vossler, K., Spitzer, L., y Hatzfeld, H., Introducción a la estilística romance. Buenos Aires: Instituto de Filología, pp. 91-148.

SPITZER, L. 1968. Lingüística e historia literaria (2. ${ }^{\mathrm{a}}$ ed.) Madrid: Gredos.

VOSSLER, K. 1929. Positivismo e idealismo en la lingüística y El lenguaje como creación y evolución. Madrid-Buenos Aires: Poblet.

VOSSLER, K. 1955. Cultura y lengua de Francia. Buenos Aires: Losada.

VOSSLER, K. 1963. Filosofía del lenguaje (4. ${ }^{\mathrm{a}}$ ed.) Buenos Aires: Losada.

YLLERA, A. 1979. Estilística, poética y semiótica literaria. Madrid: Alianza Editorial.

\section{ANEXOS}

Anexo 1. Características sintácticas "Elisa”

\begin{tabular}{|c|c|c|c|c|c|}
\hline $\mathrm{CO}$ & CÓDIGO NUMÉRICO & TIPO ORAC. & NÚC. PRED. & $\begin{array}{l}\text { FUNCIÓN } \\
\text { SUBORD. }\end{array}$ & NEXO SUBORD. \\
\hline 1 & 110221 & $\mathrm{ub}$ & $\mathrm{vV}$ & $0 \mathrm{cs}$ & $0 \mathrm{qu}$ \\
\hline 2 & 110 & $\mathrm{u}$ & $\mathrm{v}$ & 0 & 0 \\
\hline 3 & 110 & $\mathrm{u}$ & $\mathrm{s}$ & 0 & 0 \\
\hline 4 & 110221332 & $\mathrm{bbb}$ & vvi & $0 \mathrm{cscc}$ & 0 qu a \\
\hline 5 & 110 & $\mathrm{~b}$ & $\mathrm{v}$ & 0 & 0 \\
\hline 6 & $\begin{array}{l}11022130302433546 \\
060376\end{array}$ & $\mathrm{bb0b} 0 \mathrm{~b}$ & $\mathrm{vg} 0 \mathrm{vi} 0 \mathrm{v}$ & $0 \mathrm{~m} 0 \mathrm{cst} 0 \mathrm{cs}$ & $000 \mathrm{qu}$ al $0 \mathrm{qu}$ \\
\hline 7 & 110 & $\mathrm{u}$ & $\mathrm{s}$ & 0 & 0 \\
\hline 8 & 110 & $\mathrm{~b}$ & $\mathrm{v}$ & 0 & 0 \\
\hline 9 & 1102213030 & u b 0 & s v 0 & $0 \operatorname{cs~} 0$ & $0 \mathrm{dd} 0$ \\
\hline 10 & 212120 & $\mathrm{bb}$ & $\mathrm{p}(\mathrm{v})$ & $\mathrm{mo}$ & 00 \\
\hline 11 & 110 & $\mathrm{u}$ & $\mathrm{s}$ & 0 & 0 \\
\hline 12 & 110 & $\mathrm{u}$ & $\mathrm{s}$ & 0 & 0 \\
\hline 13 & 110 & $\mathrm{u}$ & $\mathrm{s}$ & 0 & 0 \\
\hline 14 & 110 & $\mathrm{~b}$ & $\mathrm{v}$ & 0 & 0 \\
\hline 15 & 110 & $\mathrm{~b}$ & $\mathrm{v}$ & 0 & 0 \\
\hline 16 & 110 & $\mathrm{~b}$ & (v) & 0 & 0 \\
\hline
\end{tabular}




\begin{tabular}{|c|c|c|c|c|c|}
\hline 17 & 110 & $\mathrm{~b}$ & $\mathrm{v}$ & 0 & 0 \\
\hline 18 & 110 & $\mathrm{~b}$ & $\mathrm{v}$ & 0 & 0 \\
\hline 19 & 110221 & $\mathrm{bb}$ & $\mathrm{V} \mathrm{v}$ & $0 \mathrm{~d}$ & 0 que \\
\hline 20 & 110221 & $\mathrm{bb}$ & $\mathrm{V} \mathrm{v}$ & $0 \mathrm{~d}$ & 0 como \\
\hline 21 & 110221 & $\mathrm{bb}$ & $\mathrm{v} \mathrm{v}$ & $0 \mathrm{cs}$ & $0 \mathrm{qu}$ \\
\hline 22 & 110 & $\mathrm{~b}$ & $\mathrm{v}$ & 0 & 0 \\
\hline 23 & 110 & $\mathrm{~b}$ & $\mathrm{v}$ & 0 & 0 \\
\hline 24 & 110 & $\mathrm{~b}$ & $\mathrm{v}$ & 0 & 0 \\
\hline 25 & 110 & $\mathrm{~b}$ & $\mathrm{v}$ & 0 & 0 \\
\hline 26 & 120231241354 & $\mathrm{bbbb}$ & $\mathrm{ad}(\mathrm{v})(\mathrm{v}) \mathrm{v}$ & $0 \mathrm{~m} \mathrm{~m} \mathrm{cs}$ & $000 \mathrm{qu}$ \\
\hline 27 & 110221332 & $\mathrm{bbu}$ & vi v & $0 \mathrm{f} \mathrm{cp}$ & 0 para qu \\
\hline 28 & 110 & $\mathrm{~b}$ & $\mathrm{ad}$ & 0 & 0 \\
\hline 29 & 212120 & $\mathrm{bb}$ & iv & t 0 & al 0 \\
\hline 30 & 2121202324040 & $\mathrm{~b} b \mathrm{~b} 0$ & v v v 0 & t $0 \operatorname{cs~} 0$ & mtras 0 qu 0 \\
\hline 31 & $: 112120$ & u b & $\mathrm{s} \mathrm{v}$ & d 0 & 00 \\
\hline 32 & 212120 & $\mathrm{~b} \mathrm{~b}$ & $(\mathrm{v}) \mathrm{v}$ & com 0 & igual-q 0 \\
\hline 33 & 110221 & $\mathrm{bb}$ & $\mathrm{vi}$ & $0 \mathrm{~s}$ & 00 \\
\hline 34 & $\begin{array}{l}11022130302435050 \\
265376386\end{array}$ & bb0b0bbb & v p 0v0 ivv & $0 \mathrm{~m} 0 \mathrm{cs} 0 \mathrm{fcs} c \mathrm{ss}$ & 000 qu 0 a qu (qu) \\
\hline 35 & 110 & $\mathrm{~b}$ & $\mathrm{ad}$ & 0 & 0 \\
\hline 36 & 110 & $\mathrm{~b}$ & $\mathrm{v}$ & 0 & 0 \\
\hline 37 & 110 & $\mathrm{~b}$ & $\mathrm{v}$ & 0 & 0 \\
\hline 38 & 213223130 & $\mathrm{bbb}$ & i iv & $\mathrm{m} \mathrm{m} 0$ & $\sin \sin 0$ \\
\hline 39 & 1102215332443050 & $\mathrm{~b} \mathrm{~b} \mathrm{~b} \mathrm{~b} 0$ & vvvi0 & 0 com $t$ cs 0 & 0 com cdo $\sin 0$ \\
\hline 40 & 110 & $\mathrm{~b}$ & $\mathrm{v}$ & 0 & 0 \\
\hline 41 & 110221332 & $\mathrm{bbu}$ & $\mathrm{v}(\mathrm{v}) \mathrm{v}$ & $0 \operatorname{com~t}$ & $0 \operatorname{com} 0$ \\
\hline 42 & 110 & $\mathrm{~b}$ & $\mathrm{a}$ & 0 & 0 \\
\hline 43 & 110221231343 & $\mathrm{bbbb}$ & viiv & $0 \mathrm{cscs} c p$ & 0 de de qu \\
\hline 44 & $\begin{array}{l}110221332443453 \\
4637070\end{array}$ & b b b b b b 0 & v i v g g g 0 & $0 \mathrm{cr} c s \mathrm{~m} \mathrm{~m} \mathrm{~m} 0$ & 0 a qu 0000 \\
\hline 45 & 110221 & $\mathrm{ub}$ & $\mathrm{s} \mathrm{p}$ & $0 \mathrm{cs}$ & 00 \\
\hline 46 & 110 & $\mathrm{~b}$ & $\mathrm{p}$ & 0 & 0 \\
\hline 47 & 110 & $\mathrm{~b}$ & $\mathrm{v}$ & 0 & 0 \\
\hline 48 & 110 & $\mathrm{u}$ & $\mathrm{s}$ & 0 & 0 \\
\hline 49 & $\begin{array}{l}1102213030243354 \\
465 \text { 374 487 598 3A4 } \\
\text { 4BA 3C4 3D4 3E4 3F4 } \\
\text { 3G4 4HG 5IH :3JG 4KJ } \\
\text { 3LG }\end{array}$ & $\begin{array}{l}\text { u b } 0 \text { b b b b b b } \\
\text { b b b b b b b b b } \\
\text { b b b }\end{array}$ & $\begin{array}{l}\mathrm{s} v 0 \mathrm{v} \mathrm{v} \text { a } \\
\text { pr } \mathrm{vav}(\mathrm{v}) \mathrm{v} \\
\mathrm{v} \mathrm{v} \text { i v v v }\end{array}$ & $\begin{array}{l}0 \operatorname{cs} 0 \operatorname{cst} t \mathrm{tedt} \\
\operatorname{cct} t \mathrm{tt} \csc \mathrm{cs} t \\
\mathrm{~d}\end{array}$ & $\begin{array}{l}0 \text { qu } 0 \text { qu cdo q cdo } \\
\text { q } 0 \text { cdo a cdo (cdo) } \\
\text { cdo (cdo) (cdo) qu } \\
\text { de } 0 \text { mtras-tto } 0\end{array}$ \\
\hline 50 & 110 & $\mathrm{u}$ & $\mathrm{s}$ & 0 & 0 \\
\hline 51 & 110 & $\mathrm{~b}$ & $\mathrm{v}$ & 0 & 0 \\
\hline 52 & 110221 & $\mathrm{bb}$ & $\mathrm{vi}$ & $0 \mathrm{~d}$ & 00 \\
\hline 53 & 110221332443554 & $\mathrm{ubb} b \mathrm{~b}$ & $\mathrm{sg}(\mathrm{v}) \mathrm{vi}$ & $0 \operatorname{cscom} \operatorname{cst}$ & 00 com qu al \\
\hline 54 & 110 & $\mathrm{u}$ & $\mathrm{pr}$ & 0 & 0 \\
\hline 55 & 110221 & $\mathrm{bb}$ & g i & $0 \mathrm{cc}$ & o a \\
\hline 56 & 110221 & $\mathrm{bb}$ & $\mathrm{V} \mathrm{V}$ & $0 \mathrm{cs}$ & $0 \mathrm{qu}$ \\
\hline 57 & 110 & $\mathrm{u}$ & $\mathrm{s}$ & 0 & 0 \\
\hline 58 & 110 & $\mathrm{u}$ & $\mathrm{s}$ & 0 & 0 \\
\hline 59 & 110 & $\mathrm{u}$ & $\mathrm{s}$ & 0 & 0 \\
\hline 60 & 110 & $\mathrm{u}$ & $\mathrm{s}$ & 0 & 0 \\
\hline
\end{tabular}




\begin{tabular}{|c|c|c|c|c|c|}
\hline 61 & $\begin{array}{ll}1102213325443050 \\
365476587\end{array}$ & ubbb0bbb & s v vi $0 \mathrm{v} \mathrm{v} \mathrm{v}$ & $\begin{array}{l}0 \text { com cs t } 0 \text { cs d } \\
\mathrm{cp}\end{array}$ & \begin{tabular}{|l|}
0 com qu al $0 \quad$ qu \\
q qu
\end{tabular} \\
\hline 62 & 1102213030243 & $\mathrm{ub} 0 \mathrm{~b}$ & $\mathrm{~s} \mathrm{v} 0 \mathrm{v}$ & $0 \operatorname{cs~} 0 \mathrm{cs}$ & 0 qu 0 qu \\
\hline 63 & 110 & $\mathrm{~b}$ & $\mathrm{v}$ & 0 & 0 \\
\hline 64 & 110 & $\mathrm{~b}$ & $\mathrm{v}$ & 0 & 0 \\
\hline 65 & 110221332342 & $\mathrm{~b} b \mathrm{bb}$ & $\mathrm{v} \mathrm{v} \mathrm{v} \mathrm{v}$ & $0 \mathrm{~m} \mathrm{cs} \mathrm{cs}$ & 0 sin-q qu (qu) \\
\hline 66 & 110 & $\mathrm{~b}$ & $\mathrm{~V}$ & 0 & 0 \\
\hline 67 & $\begin{array}{l}110221332342454 \\
565\end{array}$ & $\mathrm{bbbbbb}$ & $\mathrm{v}(\mathrm{v}) \mathrm{V} \mathrm{V} \mathrm{v} \mathrm{V}$ & 0 com s s cp d & $\begin{array}{l}0 \text { com qui (qui) qu } \\
\text { que }\end{array}$ \\
\hline 68 & 110 & $\mathrm{u}$ & $\mathrm{pr}$ & 0 & 0 \\
\hline 69 & 110 & $\mathrm{u}$ & $\mathrm{s}$ & 0 & 0 \\
\hline 70 & 110 & $\mathrm{u}$ & (v) & 0 & 0 \\
\hline 71 & 110221231343 & $\mathrm{bbbb}$ & v v v g & $0 \mathrm{cp} \mathrm{cp} \mathrm{m}$ & $0 \mathrm{qu}(\mathrm{qu}) 0$ \\
\hline 72 & 110221 & $\mathrm{ub}$ & pr v & $0 \mathrm{cp} \mathrm{cp} \mathrm{m}$ & $0 \mathrm{qu}$ \\
\hline 73 & 110 & $\mathrm{u}$ & $\mathrm{pr}$ & 0 & 0 \\
\hline 74 & 110 & $\mathrm{u}$ & $\mathrm{s}$ & 0 & 0 \\
\hline 75 & 110 & $\mathrm{~b}$ & $\mathrm{~g}$ & 0 & 0 \\
\hline 76 & 110221 & $\mathrm{~b} \mathrm{~b}$ & $\mathrm{v} \mathrm{v}$ & $0 \mathrm{t}$ & 0 mtras \\
\hline 77 & 110221231343 & $\mathrm{bbbb}$ & $\mathrm{vgg} \mathrm{v}$ & $0 \mathrm{~m} \mathrm{~m}$ com & $000 \mathrm{com}$ \\
\hline 78 & 110221 & $\mathrm{bb}$ & $\mathrm{v}(\mathrm{v})$ & $0 \mathrm{~d}$ & $0 \mathrm{si}$ \\
\hline 79 & 110221 & $\mathrm{bb}$ & $\mathrm{vp}$ & $0 \mathrm{~m}$ & 00 \\
\hline 80 & 110 & $\mathrm{~b}$ & $\mathrm{v}$ & 0 & 0 \\
\hline 81 & 110221 & $\mathrm{bb}$ & v v & 0 com & 0 tal-y-com \\
\hline 82 & 110221332 & $\mathrm{bbb}$ & v v g & $0 \mathrm{t} \mathrm{m}$ & 0 mtras 0 \\
\hline 83 & 110221332 & $\mathrm{ubb}$ & s pr v & $0 \mathrm{cs} \mathrm{c}$ & 0 qu pq \\
\hline 84 & 110221231 & $\mathrm{bbb}$ & $\mathrm{vpg}$ & $0 \mathrm{~m} \mathrm{~m}$ & 000 \\
\hline 85 & 110 & $\mathrm{~b}$ & $\mathrm{v}$ & 0 & 0 \\
\hline 86 & 110 & $\mathrm{~b}$ & $\mathrm{v}$ & 0 & 0 \\
\hline 87 & 110 & $\mathrm{~b}$ & $\mathrm{v}$ & 0 & 0 \\
\hline 88 & 110 & $\mathrm{~b}$ & $\mathrm{v}$ & 0 & 0 \\
\hline 89 & 110 & $\mathrm{~b}$ & $\mathrm{~V}$ & 0 & 0 \\
\hline 90 & 110 & $\mathrm{~b}$ & (v) & 0 & 0 \\
\hline 91 & 110221231 & $\mathrm{bbb}$ & v v v & $0 \mathrm{dc}$ & 0 que pq \\
\hline 92 & 110221 & $\mathrm{bb}$ & $(\mathrm{v}) \mathrm{v}$ & $0 \mathrm{cp}$ & $0 \mathrm{qu}$ \\
\hline 93 & 110 & $\mathrm{~b}$ & (v) & 0 & 0 \\
\hline 94 & 110 & $\mathrm{~b}$ & $\mathrm{v}$ & 0 & 0 \\
\hline 95 & 110 & $\mathrm{u}$ & $\mathrm{s}$ & 0 & 0 \\
\hline 96 & 110 & $\mathrm{~b}$ & $\mathrm{v}$ & 0 & 0 \\
\hline 97 & 110 & $\mathrm{~b}$ & $\mathrm{v}$ & 0 & 0 \\
\hline 98 & 212120 & $\mathrm{bb}$ & (v) $\mathrm{v}$ & com 0 & com 0 \\
\hline 99 & 110221 & $\mathrm{bb}$ & $\mathrm{V} \mathrm{v}$ & $0 \mathrm{~d}$ & $0 \mathrm{q}$ \\
\hline 100 & 110221 & $\mathrm{bb}$ & $\mathrm{V} \mathrm{V}$ & $0 \mathrm{~s}$ & $0 \mathrm{q}$ \\
\hline 101 & 110 & $\mathrm{~b}$ & $\mathrm{v}$ & 0 & 0 \\
\hline 102 & 110 & $\mathrm{~b}$ & $\mathrm{~V}$ & 0 & 0 \\
\hline 103 & 110 & $\mathrm{~b}$ & $\mathrm{v}$ & 0 & 0 \\
\hline 104 & 110 & $\mathrm{u}$ & $\mathrm{v}$ & 0 & 0 \\
\hline 105 & 110 & $\mathrm{u}$ & $\mathrm{pr}$ & 0 & 0 \\
\hline 106 & 110 & $\mathrm{~b}$ & $\mathrm{~g}$ & 0 & 0 \\
\hline 107 & 110 & $\mathrm{u}$ & $\mathrm{pr}$ & 0 & 0 \\
\hline 108 & 110221 & $\mathrm{bb}$ & $\mathrm{g} \mathrm{v}$ & $0 \mathrm{~d}$ & $0 \mathrm{q}$ \\
\hline
\end{tabular}




\begin{tabular}{|c|c|c|c|c|c|}
\hline 109 & 110 & $\mathrm{~b}$ & $\mathrm{~g}$ & 0 & 0 \\
\hline 110 & 110 & $\mathrm{~b}$ & $\mathrm{~g}$ & 0 & 0 \\
\hline 111 & 110 & $\mathrm{~b}$ & $\mathrm{~g}$ & 0 & 0 \\
\hline 112 & 110 & $\mathrm{u}$ & $\mathrm{s}$ & 0 & 0 \\
\hline 113 & 110 & $\mathrm{u}$ & $\mathrm{s}$ & 0 & 0 \\
\hline 114 & 110221 & $\mathrm{ub}$ & $\mathrm{s} \mathrm{v}$ & $0 \mathrm{cs}$ & $0 \mathrm{qu}$ \\
\hline 115 & 110 & $\mathrm{u}$ & $\mathrm{s}$ & 0 & 0 \\
\hline 116 & 110 & $\mathrm{u}$ & $\mathrm{s}$ & 0 & 0 \\
\hline 117 & 110 & $\mathrm{u}$ & $\mathrm{s}$ & 0 & 0 \\
\hline 118 & 110221 & $\mathrm{ub}$ & $\mathrm{sg}$ & $0 \mathrm{cs}$ & 00 \\
\hline 119 & 110221 & $\mathrm{ub}$ & $\mathrm{s} \mathrm{v}$ & $0 \mathrm{cs}$ & $0 \mathrm{qu}$ \\
\hline 120 & 110221 & $\mathrm{u} \mathrm{b}$ & $\mathrm{sv}$ & $0 \mathrm{cs}$ & $0 \mathrm{qu}$ \\
\hline 121 & 110221 & $\mathrm{ub}$ & $\mathrm{sv}$ & $0 \mathrm{cs}$ & $0 \mathrm{qu}$ \\
\hline 122 & 1102213324040354 & $\mathrm{ubb0b}$ & prvg $0 \mathrm{~g}$ & $0 \mathrm{cp} \mathrm{m} 0 \mathrm{~m}$ & 0 qu 0000 \\
\hline 123 & 110221231343 & $\mathrm{bbbb}$ & vgg v & $0 \mathrm{~m} \mathrm{~m} \mathrm{cp}$ & $000 \mathrm{qu}$ \\
\hline 124 & 110 & $\mathrm{~b}$ & $\mathrm{v}$ & 0 & 0 \\
\hline 125 & 110 & $\mathrm{~b}$ & $\mathrm{v}$ & 0 & 0 \\
\hline 126 & 110 & $\mathrm{~b}$ & $\mathrm{v}$ & 0 & 0 \\
\hline 127 & 110221332 & $\mathrm{bbb}$ & v v v & $0 \mathrm{~d} \mathrm{t}$ & 0 como cdo \\
\hline 128 & 110 & $\mathrm{~b}$ & $\mathrm{v}$ & 0 & 0 \\
\hline 129 & 110 & $\mathrm{~b}$ & $\mathrm{v}$ & 0 & 0 \\
\hline 130 & 110 & $\mathrm{~b}$ & $\mathrm{v}$ & 0 & 0 \\
\hline 131 & $\begin{array}{l}110221332443554 \\
665675787\end{array}$ & $\mathrm{bbbbbbbb}$ & v vi pr v g g v & $0 \mathrm{cs} \mathrm{d} \mathrm{d} \mathrm{cp} \mathrm{m} \mathrm{m} \mathrm{d}$ & 0 qu 0 q qu 00 q \\
\hline 132 & 110 & $\mathrm{~b}$ & $\mathrm{v}$ & 0 & 0 \\
\hline 133 & -110 & $\mathrm{~b}$ & $\mathrm{v}$ & 0 & 0 \\
\hline 134 & -110 & $\mathrm{~b}$ & (v) & 0 & 0 \\
\hline 135 & -110 & $\mathrm{~b}$ & (v) & 0 & 0 \\
\hline 136 & 110 & $\mathrm{~b}$ & $\mathrm{v}$ & 0 & 0 \\
\hline 137 & 1102213030243253 & bu 0 b b & $\mathrm{v} \mathrm{v} 0 \mathrm{v} \mathrm{v}$ & $0 \mathrm{t} 0 \mathrm{tt}$ & $\begin{array}{l}0 \text { desde } 0 \text { desde-q } \\
\text { desde-q }\end{array}$ \\
\hline 138 & 110221 & $\mathrm{bb}$ & $\mathrm{v} \mathrm{v}$ & $0 \mathrm{~d}$ & 0 que \\
\hline 139 & 110 & $\mathrm{~b}$ & $\mathrm{~V}$ & 0 & 0 \\
\hline 140 & 110 & $\mathrm{~b}$ & $\mathrm{v}$ & 0 & 0 \\
\hline 141 & 110 & $\mathrm{~b}$ & $\mathrm{v}$ & 0 & 0 \\
\hline 142 & -110 & $\mathrm{~b}$ & $(\mathrm{v})$ & 0 & 0 \\
\hline 143 & -110 & $\mathrm{~b}$ & (v) & 0 & 0 \\
\hline 144 & 110 & $\mathrm{~b}$ & $\mathrm{v}$ & 0 & 0 \\
\hline 145 & 110 & $\mathrm{u}$ & $\mathrm{s}$ & 0 & 0 \\
\hline 146 & 110 & $\mathrm{~b}$ & $\mathrm{v}$ & 0 & 0 \\
\hline 147 & 110 & $\mathrm{~b}$ & $\mathrm{~V}$ & 0 & 0 \\
\hline 148 & 110 & $b$ & $\mathrm{v}$ & 0 & 0 \\
\hline 149 & 110 & $\mathrm{u}$ & $\mathrm{s}$ & 0 & 0 \\
\hline 150 & 110 & $\mathrm{~b}$ & $\mathrm{v}$ & 0 & 0 \\
\hline 151 & 110 & $\mathrm{~b}$ & $\mathrm{~V}$ & 0 & 0 \\
\hline 152 & 110 & $b$ & $\mathrm{v}$ & 0 & 0 \\
\hline 153 & 110 & $\mathrm{~b}$ & $\mathrm{v}$ & 0 & 0 \\
\hline 154 & -110 & $\mathrm{~b}$ & $\mathrm{v}$ & 0 & 0 \\
\hline 155 & -110 & $\mathrm{~b}$ & (v) & 0 & 0 \\
\hline 156 & 110 & $b$ & $\mathrm{v}$ & 0 & 0 \\
\hline
\end{tabular}




\begin{tabular}{|c|c|c|c|c|c|}
\hline 157 & 110221 & $\mathrm{bb}$ & a v & $0 \mathrm{c}$ & $0 \mathrm{pq}$ \\
\hline 158 & 110221332443554 & $\mathrm{bbbbb}$ & vpvvi & $0 \mathrm{~m} \mathrm{c} \mathrm{m} \mathrm{cs}$ & $00 \mathrm{de}-\mathrm{q}$ com de \\
\hline 159 & 110 & $\mathrm{~b}$ & $\mathrm{v}$ & 0 & 0 \\
\hline 160 & 110 & $\mathrm{~b}$ & $\mathrm{v}$ & 0 & 0 \\
\hline 161 & 110 & $\mathrm{~b}$ & $\mathrm{v}$ & 0 & 0 \\
\hline 162 & 110 & $\mathrm{~b}$ & $\mathrm{v}$ & 0 & 0 \\
\hline 163 & 110221 & $\mathrm{bb}$ & $\mathrm{v} \mathrm{v}$ & $0 \mathrm{~s}$ & 0 que \\
\hline 164 & 110221 & $\mathrm{bb}$ & $\mathrm{vi}$ & $0 \mathrm{f}$ & o a \\
\hline 165 & 110 & $\mathrm{~b}$ & $\mathrm{v}$ & 0 & 0 \\
\hline 166 & 110 & $\mathrm{~b}$ & $\mathrm{v}$ & 0 & 0 \\
\hline 167 & 110221332 & $\mathrm{bbb}$ & $\mathrm{VvV}$ & $0 \mathrm{dt}$ & $0 \mathrm{q}$ mtras \\
\hline 168 & 110221332 & $\mathrm{bbb}$ & viv & $0 \mathrm{dt}$ & $00 \mathrm{mtras}$ \\
\hline 169 & 110221 & $\mathrm{bb}$ & $\mathrm{vv}$ & $0 \mathrm{t}$ & 0 cdo \\
\hline 170 & $\begin{array}{l}110221231241251 \\
365271281\end{array}$ & b b b b b ubb & V V V V V V V V & $0 \mathrm{ddddtdd}$ & 0 q q q q 0 q q \\
\hline 171 & 110221332443 & $\mathrm{bbbb}$ & V v s v & $0 \mathrm{c} \mathrm{d} \mathrm{cs}$ & $0 \mathrm{pq} \mathrm{q} \mathrm{qu}$ \\
\hline 172 & 110 & $\mathrm{u}$ & $\mathrm{s}$ & 0 & 0 \\
\hline 173 & 110 & $\mathrm{~b}$ & $\mathrm{v}$ & 0 & 0 \\
\hline 174 & 110 & $\mathrm{~b}$ & $\mathrm{v}$ & 0 & 0 \\
\hline 175 & 110 & $\mathrm{~b}$ & $\mathrm{v}$ & 0 & 0 \\
\hline 176 & 110221 & $\mathrm{bb}$ & $\mathrm{v}(\mathrm{v})$ & $0 \mathrm{t}$ & 0 mtras-tto \\
\hline 177 & 110 & $\mathrm{~b}$ & $\mathrm{v}$ & 0 & 0 \\
\hline 178 & 110221 & $\mathrm{bb}$ & $\mathrm{vv}$ & $0 \mathrm{~d}$ & $0 \mathrm{q}$ \\
\hline 179 & 110 & $\mathrm{~b}$ & $\mathrm{v}$ & 0 & 0 \\
\hline 180 & 212120 & $\mathrm{bb}$ & $\mathrm{pv}$ & $\mathrm{mo}$ & 00 \\
\hline 181 & 110 & $\mathrm{~b}$ & $\mathrm{v}$ & 0 & 0 \\
\hline 182 & 110 & $\mathrm{~b}$ & $\mathrm{v}$ & 0 & 0 \\
\hline 183 & 110 & $\mathrm{~b}$ & $\mathrm{v}$ & 0 & 0 \\
\hline 184 & 11022130302435050 & $\mathrm{~b} b 0 \mathrm{~b} 0$ & $\operatorname{vg} 0 \mathrm{i} 0$ & $0 \mathrm{~m} 0 \mathrm{~d} 0$ & 0000000 \\
\hline
\end{tabular}

Anexo 2. Tipos estructurales de conjuntos oracionales presentes en "Elisa"

\begin{tabular}{|l|c|c|}
\hline \multicolumn{1}{|c|}{ ESTRUCTURA } & CANTIDAD & \% \\
\hline-110 & 7 & 3,80 \\
\hline$: 112120$ & 1 & 0,54 \\
\hline 110 & 106 & 57,61 \\
\hline 110221 & 30 & 1,63 \\
\hline 110221231 & 2 & 1,09 \\
\hline 110221231241251365271281 & 1 & 0,54 \\
\hline 110221231343 & 4 & 2,17 \\
\hline 110221332 & 8 & 4,35 \\
\hline 110221332342 & 1 & 0,54 \\
\hline 110221332342454565 & 1 & 0,54 \\
\hline 110221332443 & 1 & 0,54 \\
\hline 1102213324434534637070 & 2 & 0,54 \\
\hline 110221332443554 & 1 & 1,09 \\
\hline 110221332443554665675787 & 1 & 0,54 \\
\hline 1102213324040354 & & 0,54 \\
\hline
\end{tabular}




\begin{tabular}{|l|l|l|}
\hline 1102213325443050365476587 & 1 & 0,54 \\
\hline 1102213030 & 1 & 0,54 \\
\hline 1102213030243 & 1 & 0,54 \\
\hline 1102213030243253 & 1 & 0,54 \\
\hline $\begin{array}{l}1102213030243 \text { 354 465 374 487 598 3A4 4BA 3C4 } \\
\text { 3D4 3E4 3F4 3G4 4HG 5IH :3JG 4KJ 3LG }\end{array}$ & 1 & 0,54 \\
\hline 11022130302433546060376 & 1 & 0,54 \\
\hline 11022130302435050 & 1 & 0,54 \\
\hline 11022130302435050265376386 & 1 & 0,54 \\
\hline 1102215332443050 & 1 & 0,54 \\
\hline 110231241354 & 1 & 0,54 \\
\hline 212120 & 1 & 2,72 \\
\hline 2121202324040 & 1 & 0,54 \\
\hline 213223130 & & 0,54 \\
\hline
\end{tabular}

Anexo 3. Características sintácticas "Miedo"

\begin{tabular}{|c|c|c|c|c|c|}
\hline $\mathrm{CO}$ & CÓDIGO NUMÉRICO & TIPO ORAC. & NÚC. PRED. & $\begin{array}{l}\text { FUNCIÓN } \\
\text { SUBORD. }\end{array}$ & NEXO SUBORD. \\
\hline 1 & 110221 & $\mathrm{~b} \mathrm{~b}$ & $\mathrm{vi}$ & $0 \mathrm{f}$ & 0 para \\
\hline 2 & 1102213030 & b u 0 & $\mathrm{~s} \vee 0$ & $0 \operatorname{cs~} 0$ & 0 qu 0 \\
\hline 3 & 110 & $\mathrm{~b}$ & $\mathrm{v}$ & 0 & 0 \\
\hline 4 & 110 & $\mathrm{~b}$ & $\mathrm{~s}$ & 0 & 0 \\
\hline 5 & 110 & $\mathrm{~b}$ & $\mathrm{v}$ & 0 & 0 \\
\hline 6 & 1102212313435050 & $\mathrm{~b} \mathrm{~b} \mathrm{~b} \mathrm{~b} 0$ & $\mathrm{v} \mathrm{v} v(\mathrm{v}) 0$ & 0 cs cs com 0 & $0 \mathrm{qu}(\mathrm{qu}) \operatorname{com} 0$ \\
\hline 7 & 110221 & $\mathrm{bb}$ & $\mathrm{si}$ & $0 \mathrm{cs}$ & $0 \mathrm{de}$ \\
\hline 8 & 110221332 & $\mathrm{bbb}$ & $\mathrm{vvi}$ & $0 \mathrm{dc}$ & 0 q por \\
\hline 9 & 110221 & $\mathrm{bb}$ & $\mathrm{a}(\mathrm{a})$ & $0 \mathrm{com}$ & $0 \mathrm{com}$ \\
\hline 10 & 110 & $\mathrm{~b}$ & $\mathrm{v}$ & 0 & 0 \\
\hline 11 & 110221 & $\mathrm{~b} \mathrm{~b}$ & $\mathrm{vv}$ & $0 \mathrm{cs}$ & $0 \mathrm{qu}$ \\
\hline 12 & 110 & $\mathrm{~b}$ & $\mathrm{v}$ & 0 & 0 \\
\hline 13 & 110221 & $\mathrm{~b} \mathrm{~b}$ & $\mathrm{vv}$ & 0 con & 0 aung \\
\hline 14 & 110 & $\mathrm{~b}$ & $\mathrm{v}$ & 0 & 0 \\
\hline 15 & 110 & $\mathrm{~b}$ & $\mathrm{~s}$ & 0 & 0 \\
\hline 16 & 110221332 & $\mathrm{bbb}$ & $\mathrm{vvi}$ & $0 \mathrm{cs} \mathrm{d}$ & 0 qu 0 \\
\hline 17 & 110 & $\mathrm{~b}$ & $\mathrm{v}$ & 0 & 0 \\
\hline 18 & 110 & $\mathrm{~b}$ & $\mathrm{a}$ & 0 & 0 \\
\hline 19 & 110 & $\mathrm{~b}$ & $\mathrm{v}$ & 0 & 0 \\
\hline 20 & 110 & $\mathrm{~b}$ & $\mathrm{v}$ & 0 & 0 \\
\hline 21 & 110221 & $\mathrm{~b} \mathrm{~b}$ & $\mathrm{v} \mathrm{s}$ & $0 \mathrm{~h}$ & $0 \mathrm{si}$ \\
\hline 22 & 212120 & $\mathrm{bb}$ & $\mathrm{s} \mathrm{v}$ & h 0 & si 0 \\
\hline 23 & 110221231 & $\mathrm{bbb}$ & a i i & $0 \mathrm{~s} \mathrm{~s}$ & 0000 \\
\hline 24 & 110 & $\mathrm{~b}$ & $\mathrm{a}$ & 0 & 0 \\
\hline 25 & 110221 & $\mathrm{ub}$ & $\mathrm{v} \mathrm{v}$ & $0 \mathrm{cs}$ & $0 \mathrm{qu}$ \\
\hline 26 & 110221231 & $\mathrm{bbb}$ & $\mathrm{VvV}$ & $0 \mathrm{e} \mathrm{e}$ & $0 \mathrm{q}$ así-q \\
\hline 27 & 110 & $\mathrm{~b}$ & $\mathrm{a}$ & 0 & 0 \\
\hline 28 & 110 & $\mathrm{~b}$ & $\mathrm{v}$ & 0 & 0 \\
\hline 29 & 110 & $\mathrm{~b}$ & (v) & 0 & 0 \\
\hline 30 & 110 & $\mathrm{~b}$ & $\mathrm{v}$ & 0 & 0 \\
\hline
\end{tabular}




\begin{tabular}{|c|c|c|c|c|c|}
\hline 31 & 110221332 & $\mathrm{bbb}$ & $\mathrm{v}$ or a & $0 \mathrm{c} \mathrm{com}$ & $0 \mathrm{pq}$ com \\
\hline 32 & 110 & $\mathrm{~b}$ & $\mathrm{v}$ & 0 & 0 \\
\hline 33 & 1102213030 & b b 0 & a v 0 & $0 \operatorname{cs} 0$ & 0 dd 0 \\
\hline 34 & 110 & $\mathrm{~b}$ & $\mathrm{~s}$ & 0 & 0 \\
\hline 35 & 110221 & $\mathrm{ub}$ & $\mathrm{v} \mathrm{v}$ & $0 \mathrm{cs}$ & $0 \mathrm{dd}$ \\
\hline 36 & 110 & $\mathrm{u}$ & $\mathrm{pr}$ & 0 & 0 \\
\hline 37 & 110 & $\mathrm{~b}$ & $\mathrm{v}$ & 0 & 0 \\
\hline 38 & 110221 & $\mathrm{bb}$ & $\mathrm{v}(\mathrm{v})$ & $0 \mathrm{~d}$ & 0 que \\
\hline 39 & 110 & $\mathrm{~b}$ & $\mathrm{v}$ & 0 & 0 \\
\hline 40 & 110 & $\mathrm{~b}$ & $\mathrm{v}$ & 0 & 0 \\
\hline 41 & 110 & $\mathrm{~b}$ & $\mathrm{a}$ & 0 & 0 \\
\hline 42 & 110 & $\mathrm{u}$ & $\mathrm{s}$ & 0 & 0 \\
\hline 43 & 110 & $\mathrm{~b}$ & $\mathrm{i}$ & 0 & 0 \\
\hline 44 & 110 & $\mathrm{~b}$ & $\mathrm{i}$ & 0 & 0 \\
\hline 45 & 110 & $\mathrm{~b}$ & $\mathrm{v}$ & 0 & 0 \\
\hline 46 & 110221 & $\mathrm{bb}$ & $\mathrm{vi}$ & $0 \mathrm{f}$ & $0 \mathrm{a}$ \\
\hline 47 & 110 & $\mathrm{~b}$ & $\mathrm{v}$ & 0 & 0 \\
\hline 48 & 110 & $\mathrm{~b}$ & (v) & 0 & 0 \\
\hline 49 & 110221332342454 & $\mathrm{bbbbb}$ & v prvvi & $0 \mathrm{crcscst}$ & 0 con qu (qu) hasta \\
\hline 50 & 110 & $\mathrm{~b}$ & $\mathrm{v}$ & 0 & 0 \\
\hline 51 & 110 & $\mathrm{~b}$ & $\mathrm{v}$ & 0 & 0 \\
\hline 52 & 110 & $\mathrm{u}$ & $\mathrm{v}$ & 0 & 0 \\
\hline 53 & 110 & $\mathrm{u}$ & $\mathrm{v}$ & 0 & 0 \\
\hline 54 & 214224332140 & $\mathrm{bbbb}$ & v viv & $\mathrm{h} \mathrm{h}$ cs 0 & si (si) para 0 \\
\hline 55 & 110 & $\mathrm{~b}$ & $\mathrm{v}$ & 0 & 0 \\
\hline 56 & 110 & $\mathrm{~b}$ & $\mathrm{v}$ & 0 & 0 \\
\hline 57 & 110 & $\mathrm{~b}$ & $\mathrm{a}$ & 0 & 0 \\
\hline 58 & 110221332 & $\mathrm{bbb}$ & vi i & $0 \mathrm{f} \mathrm{d}$ & 0 para 0 \\
\hline 59 & 110221231241 & $\mathrm{bbbb}$ & vai i & $0 \mathrm{fff}$ & 0 para (para) (para) \\
\hline 60 & 110 & $\mathrm{u}$ & $\mathrm{s}$ & 0 & 0 \\
\hline 61 & 110 & $\mathrm{~b}$ & $\mathrm{v}$ & 0 & 0 \\
\hline 62 & 110 & $\mathrm{~b}$ & $\mathrm{v}$ & 0 & 0 \\
\hline 63 & 110 & $\mathrm{~b}$ & $\mathrm{v}$ & 0 & 0 \\
\hline 64 & 110 & $\mathrm{u}$ & $\mathrm{v}$ & 0 & 0 \\
\hline 65 & 110 & $\mathrm{~b}$ & $\mathrm{v}$ & 0 & 0 \\
\hline 66 & 110 & $\mathrm{~b}$ & $\mathrm{~s}$ & 0 & 0 \\
\hline 67 & 110 & $\mathrm{~b}$ & $\mathrm{a}$ & 0 & 0 \\
\hline 68 & 110 & $\mathrm{~b}$ & $\mathrm{v}$ & 0 & 0 \\
\hline 69 & 110 & $\mathrm{~b}$ & $\mathrm{v}$ & 0 & 0 \\
\hline 70 & 110221 & $\mathrm{bb}$ & $\mathrm{vs}$ & $0 \mathrm{~d}$ & $0 \mathrm{q}$ \\
\hline 71 & 110221231 & $\mathrm{bbb}$ & $\mathrm{vVv}$ & $0 \mathrm{~d} \mathrm{~d}$ & $0 \mathrm{qq}$ \\
\hline 72 & 110 & $\mathrm{~b}$ & $\mathrm{v}$ & 0 & 0 \\
\hline 73 & 110 & $\mathrm{~b}$ & $\mathrm{v}$ & 0 & 0 \\
\hline 74 & 110 & $\mathrm{~b}$ & $\mathrm{v}$ & 0 & 0 \\
\hline 75 & 110221 & $\mathrm{bb}$ & $\mathrm{vi}$ & $0 \mathrm{~d}$ & 00 \\
\hline 76 & 110221332342454464 & $\mathrm{bbbbbb}$ & v v v v v v & $0 \mathrm{dcctt}$ & $\begin{array}{l}0 \mathrm{q} \mathrm{pq}(\mathrm{pq}) \text { antes-de-q } \\
\text { (antes-de-q) }\end{array}$ \\
\hline 77 & 110 & $\mathrm{~b}$ & $\mathrm{v}$ & 0 & 0 \\
\hline 78 & 110 & $\mathrm{~b}$ & $\mathrm{v}$ & 0 & 0 \\
\hline 79 & $: 110121131$ & $\mathrm{bub}$ & $\mathrm{v}$ ad i & $0 \mathrm{~d} \mathrm{~d}$ & 0000 \\
\hline
\end{tabular}




\begin{tabular}{|c|c|c|c|c|c|}
\hline 80 & 110 & $\mathrm{~b}$ & $\mathrm{v}$ & 0 & 0 \\
\hline 81 & 110 & $b$ & $\mathrm{v}$ & 0 & 0 \\
\hline 82 & 110 & $\mathrm{~b}$ & $\mathrm{v}$ & 0 & 0 \\
\hline 83 & 110221 & $\mathrm{ub}$ & $\mathrm{vi}$ & $0 \mathrm{cs}$ & $0 \mathrm{qu}$ \\
\hline 84 & 110 & $\mathrm{~b}$ & $\mathrm{v}$ & 0 & 0 \\
\hline 85 & 110 & $\mathrm{~b}$ & $\mathrm{v}$ & 0 & 0 \\
\hline 86 & 110 & $\mathrm{~b}$ & $\mathrm{v}$ & 0 & 0 \\
\hline 87 & 110 & $\mathrm{~b}$ & $\mathrm{p}$ & 0 & 0 \\
\hline 88 & 110 & $\mathrm{~b}$ & $\mathrm{~g}$ & 0 & 0 \\
\hline 89 & 110 & $\mathrm{~b}$ & $\mathrm{~g}$ & 0 & 0 \\
\hline 90 & 110 & $\mathrm{~b}$ & $\mathrm{a}$ & 0 & 0 \\
\hline 91 & 110 & $\mathrm{u}$ & $\mathrm{v}$ & 0 & 0 \\
\hline 92 & 110 & $\mathrm{~b}$ & $\mathrm{v}$ & 0 & 0 \\
\hline 93 & $: 110121232$ & $\mathrm{bbu}$ & viv & $0 \mathrm{dc}$ & $00 \mathrm{q}$ \\
\hline 94 & 110221 & $\mathrm{~b} \mathrm{~b}$ & $\mathrm{vp}$ & $0 \mathrm{cs}$ & 00 \\
\hline 95 & 110 & $\mathrm{~b}$ & $\mathrm{v}$ & 0 & 0 \\
\hline 96 & 110 & $\mathrm{~b}$ & $\mathrm{v}$ & 0 & 0 \\
\hline 97 & 110221 & $\mathrm{~b} \mathrm{~b}$ & $\mathrm{vi}$ & $0 \mathrm{f}$ & 0 para \\
\hline 98 & 110 & $\mathrm{~b}$ & $\mathrm{v}$ & 0 & 0 \\
\hline 99 & 110 & $\mathrm{~b}$ & $\mathrm{v}$ & 0 & 0 \\
\hline 100 & 110 & $\mathrm{~b}$ & $\mathrm{v}$ & 0 & 0 \\
\hline 101 & 110221 & $\mathrm{~b} \mathrm{~b}$ & $\mathrm{vv}$ & $0 \mathrm{~d}$ & $0 \mathrm{q}$ \\
\hline 102 & 110221231343454 & $\mathrm{bbb} b \mathrm{~b}$ & $\mathrm{v} \mathrm{v} \mathrm{v} \mathrm{(v)} \mathrm{v}$ & $0 \mathrm{c} \mathrm{c} \mathrm{com} \mathrm{h}$ & $0 \mathrm{pq}(\mathrm{pq}) \mathrm{com} \mathrm{si}$ \\
\hline 103 & 110221 & $\mathrm{bb}$ & ad v & 0 com & $0 \mathrm{com}$ \\
\hline 104 & 110221 & $\mathrm{~b} \mathrm{~b}$ & $\mathrm{v} v$ & $0 \mathrm{~s}$ & $0 \mathrm{q}$ \\
\hline 105 & 110 & $\mathrm{~b}$ & $\mathrm{v}$ & 0 & 0 \\
\hline 106 & 110 & $\mathrm{~b}$ & $\mathrm{v}$ & 0 & 0 \\
\hline 107 & 110221 & $\mathrm{bb}$ & $\mathrm{vi}$ & $0 \mathrm{~d}$ & 00 \\
\hline 108 & 110 & $\mathrm{~b}$ & $\mathrm{v}$ & 0 & 0 \\
\hline 109 & 110 & $\mathrm{u}$ & $\mathrm{s}$ & 0 & 0 \\
\hline 110 & 110221332 & $\mathrm{bbb}$ & $\mathrm{vvi}$ & $0 \mathrm{cs}$ & $0 \mathrm{pq} 0$ \\
\hline 111 & 212120232242 & $\mathrm{bbbb}$ & vvii & h $0 \mathrm{ff}$ & si 0 a a \\
\hline 112 & $\begin{array}{l}110221231241: 251265 \\
2752852953 \mathrm{~A} 9\end{array}$ & $\begin{array}{l}\text { b b b b b u ubb } \\
\text { b }\end{array}$ & $\begin{array}{l}\text { vi ivvssav } \\
\text { v }\end{array}$ & $\begin{array}{l}0 \operatorname{cscsc} \mathrm{cdd} d \\
\mathrm{de}\end{array}$ & $\begin{array}{l}0 \text { de (de) pq (pq) } 0000 \\
0 \text { así-q }\end{array}$ \\
\hline 113 & 110 & $\mathrm{~b}$ & $\mathrm{v}$ & 0 & 0 \\
\hline 114 & 212120232242 & $\mathrm{bbbb}$ & (v) $v g p$ & com $0 \mathrm{~m} \mathrm{~m}$ & com 000 \\
\hline 115 & 1102213030 & $\mathrm{bb} 0$ & $\mathrm{v}(\mathrm{v}) 0$ & $0 \operatorname{com} 0$ & 0 com 0 \\
\hline 116 & 110 & $\mathrm{~b}$ & $\mathrm{v}$ & 0 & 0 \\
\hline 117 & 1102213030 & bu 0 & v v 0 & 0 t 0 & 0 apenas 0 \\
\hline 118 & 110 & $\mathrm{~b}$ & $\mathrm{v}$ & 0 & 0 \\
\hline 119 & 110 & $\mathrm{~b}$ & $\mathrm{v}$ & 0 & 0 \\
\hline 120 & 110 & $\mathrm{~b}$ & $\mathrm{v}$ & 0 & 0 \\
\hline 121 & 110 & $\mathrm{~b}$ & $\mathrm{v}$ & 0 & 0 \\
\hline 122 & 110221 & $\mathrm{~b} \mathrm{~b}$ & $\mathrm{vV}$ & $0 \mathrm{cs}$ & $0 \mathrm{qu}$ \\
\hline 123 & 110 & $\mathrm{~b}$ & $\mathrm{v}$ & 0 & 0 \\
\hline 124 & 110221 & $\mathrm{~b} \mathrm{~b}$ & $\mathrm{vV}$ & $0 \mathrm{~d}$ & $0 \mathrm{q}$ \\
\hline 125 & 110 & $\mathrm{~b}$ & $\mathrm{v}$ & 0 & 0 \\
\hline 126 & 110 & $\mathrm{~b}$ & $\mathrm{v}$ & 0 & 0 \\
\hline 127 & 110 & $\mathrm{~b}$ & $\mathrm{v}$ & 0 & 0 \\
\hline 128 & 110 & $\mathrm{~b}$ & $\mathrm{v}$ & 0 & 0 \\
\hline
\end{tabular}




\begin{tabular}{|c|c|c|c|c|c|}
\hline 129 & 212120232242 & $\mathrm{~b} \mathrm{~b} \mathrm{~b} \mathrm{~b}$ & iv v v & f $0 \mathrm{cscs}$ & para $0 \mathrm{dd}(\mathrm{dd})$ \\
\hline 130 & 110221 & $\mathrm{~b} \mathrm{~b}$ & $\mathrm{v} \mathrm{v}$ & $0 \mathrm{cs}$ & $0 \mathrm{qu}$ \\
\hline 131 & $110: 221232$ & $\mathrm{bbb}$ & v v v & 0 e d & $0 \mathrm{q} 0$ \\
\hline 132 & -110 & $\mathrm{~b}$ & (v) & 0 & 0 \\
\hline 133 & $(-110) 22(1)$ & (b) b & $(\mathrm{v}) \mathrm{v}$ & $(0) \mathrm{c}$ & $(0) \mathrm{pq}$ \\
\hline 134 & $-110-120-130-140$ & $\mathrm{ubbb}$ & int $\mathrm{v} v \mathrm{v}$ & 00000 & 00000 \\
\hline 135 & 110221 & $\mathrm{bb}$ & $\mathrm{vi}$ & $0 \mathrm{~d}$ & 00 \\
\hline 136 & 110 & $\mathrm{~b}$ & $\mathrm{v}$ & 0 & 0 \\
\hline 137 & 110 & $\mathrm{~b}$ & $\mathrm{v}$ & 0 & 0 \\
\hline 138 & 110 & $\mathrm{~b}$ & $\mathrm{v}$ & 0 & 0 \\
\hline 139 & 110 & $\mathrm{~b}$ & $\mathrm{v}$ & 0 & 0 \\
\hline 140 & 110 & $\mathrm{~b}$ & $\mathrm{v}$ & 0 & 0 \\
\hline 141 & 110 & $\mathrm{~b}$ & $\mathrm{v}$ & 0 & 0 \\
\hline 142 & 110 & $\mathrm{~b}$ & $\mathrm{v}$ & 0 & 0 \\
\hline 143 & 110 & $\mathrm{~b}$ & $\mathrm{v}$ & 0 & 0 \\
\hline 144 & 110221030 & b b 0 & v v 0 & $0 \mathrm{~d} 0$ & $0 \mathrm{q} 0$ \\
\hline 145 & 110 & $\mathrm{~b}$ & $\mathrm{v}$ & 0 & 0 \\
\hline 146 & 110 & $\mathrm{~b}$ & $\mathrm{v}$ & 0 & 0 \\
\hline 147 & 110 & $\mathrm{~b}$ & $\mathrm{v}$ & 0 & 0 \\
\hline 148 & 110 & $\mathrm{~b}$ & $\mathrm{v}$ & 0 & 0 \\
\hline 149 & 110 & $\mathrm{~b}$ & $\mathrm{v}$ & 0 & 0 \\
\hline 150 & 110 & $\mathrm{~b}$ & $\mathrm{v}$ & 0 & 0 \\
\hline 151 & 110221 & $\mathrm{~b} \mathrm{~b}$ & vi & $0 \mathrm{f}$ & $0 \mathrm{a}$ \\
\hline 152 & 110221231 & $\mathrm{bbb}$ & v s i & $0 \mathrm{cs} \mathrm{cs}$ & $0 \mathrm{de}(\mathrm{de})$ \\
\hline 153 & 110221 & $\mathrm{~b} \mathrm{~b}$ & vi & $0 \mathrm{f}$ & $0 \mathrm{a}$ \\
\hline 154 & 110221 & $\mathrm{~b} \mathrm{~b}$ & $\mathrm{~V} \mathrm{v}$ & $0 \mathrm{~d}$ & $0 \mathrm{q}$ \\
\hline 155 & 110221 & $\mathrm{~b} \mathrm{~b}$ & v v & $0 \mathrm{~d}$ & $0 \mathrm{q}$ \\
\hline 156 & 110221231343 & $\mathrm{bbbb}$ & v v vi & $0 \mathrm{~d} \mathrm{df}$ & 0 q sino-q (para) \\
\hline 157 & 110 & $\mathrm{~b}$ & $\mathrm{v}$ & 0 & 0 \\
\hline 158 & 110221 & $\mathrm{~b} \mathrm{~b}$ & $\mathrm{a}(\mathrm{v})$ & $0 \mathrm{ca}$ & 0 de-q \\
\hline 159 & 110 & $\mathrm{~b}$ & $\mathrm{v}$ & 0 & 0 \\
\hline 160 & 110 & $\mathrm{~b}$ & $\mathrm{v}$ & 0 & 0 \\
\hline 161 & 110 & $\mathrm{~b}$ & $\mathrm{v}$ & 0 & 0 \\
\hline 162 & 110 & $\mathrm{~b}$ & $\mathrm{v}$ & 0 & 0 \\
\hline 163 & 110 & $\mathrm{~b}$ & $\mathrm{v}$ & 0 & 0 \\
\hline 164 & 110221231 & $\mathrm{bbb}$ & v v v & $0 \mathrm{dd}$ & $0 q(q)$ \\
\hline 165 & 110 & $\mathrm{~b}$ & (v) & 0 & 0 \\
\hline 166 & 110 & $\mathrm{u}$ & $\mathrm{ad}$ & 0 & 0 \\
\hline 167 & 110 & $\mathrm{~b}$ & v & 0 & 0 \\
\hline 168 & 110221 & $\mathrm{bb}$ & $\mathrm{v}(\mathrm{v})$ & $0 \mathrm{com}$ & $0 \mathrm{com}$ \\
\hline 169 & 110 & $\mathrm{~b}$ & $\mathrm{v}$ & 0 & 0 \\
\hline 170 & 110 & $\mathrm{~b}$ & $\mathrm{v}$ & 0 & 0 \\
\hline 171 & 110 & $\mathrm{~b}$ & $\mathrm{v}$ & 0 & 0 \\
\hline 172 & 110 & $\mathrm{~b}$ & $\mathrm{v}$ & 0 & 0 \\
\hline 173 & 110 & $\mathrm{~b}$ & $\mathrm{v}$ & 0 & 0 \\
\hline 174 & 110221 & $\mathrm{bb}$ & $\mathrm{v}(\mathrm{v})$ & $0 \mathrm{~d}$ & 0 por-que \\
\hline 175 & 110 & $\mathrm{~b}$ & $\mathrm{v}$ & 0 & 0 \\
\hline 176 & 110 & $\mathrm{~b}$ & $\mathrm{v}$ & 0 & 0 \\
\hline 177 & 110221 & $\mathrm{bb}$ & v v & $0 \mathrm{~d}$ & $0 \mathrm{q}$ \\
\hline 178 & 110 & $\mathrm{~b}$ & $\mathrm{a}$ & 0 & 0 \\
\hline
\end{tabular}




\begin{tabular}{|l|l|l|l|l|l|}
\hline 179 & 110 & b & v & 0 & 0 \\
\hline 180 & 110221 & u b & vi & $0 \mathrm{cs}$ & 0 para \\
\hline 181 & 110221231 & b b b & or v i & $0 \mathrm{cp} \mathrm{s}$ & 0 qu 0 \\
\hline 182 & 110221 & b b & or i & 0 a & 00 \\
\hline
\end{tabular}

Anexo 4. Tipos estructurales de conjuntos oracionales presentes en "Miedo"

\begin{tabular}{|l|c|c|}
\hline \multicolumn{1}{|c|}{ ESTRUCTURA } & CANTIDAD & \% \\
\hline-110 & 1 & 0,54 \\
\hline$-110-120-130-140$ & 1 & 0,54 \\
\hline 110 & 115 & 63,18 \\
\hline$(-110) 22(1)$ & 1 & 0,54 \\
\hline$: 110121131$ & 1 & 0,54 \\
\hline$: 110121232$ & 1 & 0,54 \\
\hline $110: 221232$ & 1 & 0,54 \\
\hline 110221 & 33 & 18,13 \\
\hline 110221030 & 1 & 0,54 \\
\hline 110221231 & 6 & 3,29 \\
\hline 110221231241 & 1 & 0,54 \\
\hline $110221231241: 2512652752852953 A 9$ & 1 & 0,54 \\
\hline 110221231343 & 1 & 0,54 \\
\hline 110221231343454 & 1 & 0,54 \\
\hline 1102212313435050 & 1 & 0,54 \\
\hline 110221332 & 5 & 2,74 \\
\hline 110221332342454 & 1 & 0,54 \\
\hline 110221332342454464 & 1 & 0,54 \\
\hline 1102213030 & 4 & 2,19 \\
\hline 212120 & 1 & 0,54 \\
\hline 212120232242 & 3 & 1,64 \\
\hline 214224332140 & 1 & 0,54 \\
\hline
\end{tabular}

\section{NOTA SOBRE LA AUTORA}

Isamary Aldama Pando es investigadora en el Centro Cultural Pablo de la Torriente Brau, Cuba. 